\title{
(5) Spectra Technology
}

\section{EXPERIMENTAL SUPPORT FOR KrF LASER MODELING}

Final Report

Submitted to LOS ALAMOS NATIONAL LABORATORY

by SPECTRA TECHNOLOGY, INC

19 July 1989 


\title{
EXPERINENTAL SUPPORT FOR \\ KrF LASER YODELING
}

\author{
Final Report
}

$9-\times 65-W 1478-1$

\author{
Submitted to \\ Los Alamos National Laboratory \\ Post Office Box 990 \\ Los Alamos, New Mexico 87545
}

\author{
by \\ Spectra Technology, Inc. \\ 2755 Northup Way \\ Bellevue, Washington 88004-1485
}

\section{July 1889}

\section{DISCLAIMER}

This report was prepared as an account of work sponsored by an agency of the United States Government. Neither the United States Government nor any agency thereof, nor any of their employees, makes any warranty, express or implied, or assumes any legal liability or responsibility for the accuracy, completeness, or usefulness of any information, apparatus, product, or process disclosed, or represents that its use would not infringe privately owned rights. Reference herein to any specific commercial product, process, or service by trade name, trademark, manufacturer, or otherwise does not necessarily constitute or imply its endorsement, recommendation, or favoring by the United States Government or any agency thereof. The views and opinions of authors expressed herein do not necessarily state or reflect those of the United States Government or any agency thereof. 


\section{TABLB OF CONTENTS}

Section

Page

1 Executive Summary $1-1$

2 Extraction Measurements 2-1

2.1 Introduction 2-1

2.2 Description of Experiment 2-2

2.3 Determination of E-Beam Deposition 2-5

2.4 Experimental Results 2-14

2.5 Discussion 2-18

2.6 References 2-18

3 Gain and Transient Absorption Measurements 3-1

3.1 Introduction 3-1

3.2 Description of Experiment 3-1

3.3 Analysis Technique 3-3

3.4 Gain and Transient Absorption Results 3-7

3.5 Data Analysis 3-13

3.6 References 3-17

4 Conclusions 4-1

Appendix

A Development and Characterization of a Versatile A-1

Faraday Cup

A.1 Introduction A-1

A.2 Description of Faraday Cup Design A-2

A.3 Characterization Tests A-5

A.4 Conclusions A-20

A.5 References A-20

B E-Beam Deposition Calculation for Nonuniform B-1

Temperature Distribution

B.1 Introduction B-1

B.2 Model B-1

B.3 Analysis B-2

B.4 References B-5

C Intracavity Flux Dependent Absorption in E-Beam A-1 Pumped KrF 
2-1 Cross-section of Tahoma Laser Chamber.

2-2 Schematic of E-Beam Deposition Profile Measurement System. Not shown are lenses and a narruwband filter used between the fibers and the PMT system to isolate only the $248 \mathrm{~nm}$ light.

2-3 E-Beam Deposition Profile Results. The data points are from the fiber optic measurements; the curve is from the LANL Monte Carlo E-beam deposition code. (a) Deposition profile in horizontal direction away from the e-beam foil. (b) Deposition profile in vertical direction along the e-beam foil.

2-4 Schematic Representation of the E-beam Pumped Regions Within the Laser Chamber With the Beam Stop in Place.

2-5 Intrinsic Laser Efficiency as a Function of the Initial Krypton Concentration.

2-6 Marx Current and Laser Output Waveforms. (a) For
the Ar-diluent case. (b) For the Kr-diluent case.

$\mathrm{KrF}^{*}$ Sidelight Fluorescence Waveforms.

(a) Under nonlasing conditions. (b) Under lasing conditions. Note, approximately $50 \%$ of the light being detected is from outside the active volume.

3-1 Schematic of the Gain and Transient Absorption Measurement System.

3-3 Comparison of the Gain and Absorption Data With the LANL Kodel Predictions.

3-4 Measurements of the Off-Line Absorption as a Function of the Average Intracavity Flux.

A-1 Schematic Diagram of the STI Faraday Cup A-4

A-2 Details of the Corrugated Collector Plate Dimensions $A-6$

A-3 Equivalent Electrical Schematic for the Faraday $\quad$ A-7

A-4 Cross-section of the Tahoma Laser Chamber Showing A-8 the Typical Location for the Faraday Cup. 
A-5 Normalized Output From the Faraday Cup as $\Lambda$

$A-10$

Function of the Atomic Mass of the Collector Plate.

The curve is from Reference $(\Lambda-1)$.

A-6 Comparison of Different Collector Plates. The top trace is the Marx current obtained from a $\mathrm{dB} / \mathrm{dt}$ probe within the diode chamber; the bottom trace is the output from the Faraday Cup. Time scale is $100 \mathrm{~ns} /$ div. (a) For corrugated carbon. (b) For corrugated aluminum. (c) For flat aluminum.

A-7 Faraday Cup Linearity Check. Plotted is the cup output signal versus the geometrical transmission factor for various perforated masks placed between the e-beam and the cup.

A-8 Relative Effect of $0.025 \mathrm{~mm}$ Kapton Foil Over Collector Plate. The top trace is the Marx current obtained from a dB/dt probe within the diode chamber; the bottom trace is the output from the Faraday Cup. Time scale is $100 \mathrm{~ns} / \mathrm{div}$. The carbon face plate is used under vacuum conditions. (a) Without the foil. (b) With the foil.

A-9 Performance of the Faraday Cup in a Gas Mixture (10\% Kr/80\% Ar at 1000 Torr). The top trace is the Marx current obtained from a dB/dt probe within the diode chamber; the bottom trace is the output from the Faraday Cup. Time scale is $100 \mathrm{~ns} / \mathrm{div}$. (a) Without the Kapton foil. (b) With the Kapton foil.

A-10 Comparison of Current Density With Either Vacuum or Air in the Laser Chamber. The carbon face plate and Kapton foil are used.

A-11 Comparison of Current Density With Either Air or a $\mathrm{Kr} / \mathrm{Ar}$ Mixture in the Laser Chamber. The carbon face plate and Kapton foil are used.

A-12 Performance of Faraday Cup During Different Grounding Conditions. The top trace is the Marx current obtained from a dB/dt probe within the diode chamber; the bottom trace is the output from the Faraday Cup. Time scale is $100 \mathrm{~ns} /$ div. (a) With the cup grounded to the laser chamber in room air. (b) With the cup not grounded directly to the laser chamber in room air. (c) With the cup not grounded directly to the laser chamber in vacuum.

Schematic of Laser Chamber Showing Definitions Used in the Model Analysis. 
LIST OF TABLES

\section{Table}

2-1 Summary of Gas Mixture Compositions During Extraction Measurements

\section{3-8}

3-1 Intensity Ratio Data

ec

Gain/Loss Values

$3-16$

3-3

Gain/Loss Summary at $248.4 \mathrm{~nm}$

3-1]

30

1 in

$1 \ldots$

J lu

1

I $f_{1}$

$1=$

II $C$,

n de

$S$

d is 
detected the $\mathrm{KrF}^{*}$ fluorescence as a function of horizontal and vertical distance with respect to the e-beam foil. These results were compared with a Monte Carlo e-beam deposition code developed at LANL.

4) A versatile Faraday cup design was developed and tested. This proved to be a very useful diagnostic during this program, and we believe it will continue to be so in future work.

5) A short pulse ( $\simeq 3$ ns), variable wavelength probe laser system was used to measure the transient absorption and gain of the Tahoma KrF laser under lasing and nonlasing conditions. These results were compared with the predictions of the $\mathrm{KrF}$ model developed by LANL.

6) Saturation flux and power efficiency analysis were performed on the data. The analysis demonstrated that the data are related in a self-consistent manner.

In addition to these experimental accomplishments, the results of this program were presented at a number of conferences and workshops. These are listed below:

1) W.D. Kimura, J.F. Seamans, and D.E. Hanson, "Intracavity Flux Dependent Absorption Measurements in E-Beam Pumped KrF, "11th International Conference on Lasers '88, Lake Tahoe, NV, Dec. 58, 1888, Paper EXC-21.

2) W.D. Kimura and J.F. Seamans, "Review of Recent KrF Kinetics Experiments at STI, "Workshop on $\mathrm{KrF}$ Laser Technology, Santa Fe, NM, Apr. 18-21, 1889, Paper ThA6.

3) W.D. Kimura, J.F. Seamans, F. Kannari, and D.E. Hanson, "Comparisons of Recent E-Beam Pumped KrF Laser Experiments With Model Predictions, "Conference on Lasers and Electro-Optics (CLEO), Baltimore, MD, Apr. 24-28, 1889, Paper ThP4. 
4) J.F. Seamans and W.D. Kimura, "Characterization of a Versatile Faraday Cup Design," Conference on Lasers and Electro-Optics (CLEO), Baltimore, LD, Apr. 24-28, 1989, Paper WF16.

In summary, this program has provided LANL with data critical for their model development. Additional needed data and issues were also identified and are currently being investigated under a new contract with LANL which followed this one.

The outline of the remainder of this report is as follows. Section 2 describes the laser extraction experiments together with the calibration of the diagnostics and the re-examination of the analysis procedure. Section 3 discusses the gain and transient absorption measurements, and Section 4 gives the conclusions for this program. Characterization of the Faraday cup developed during this program is presented in the Appendix A. Appendix B gives details of the thermodynamic analysis of the e-beam deposition taking into account nonuniform temperature distributions within the laser gas chamber. Appendix $C$ is a copy of the Lasers,'88 paper.

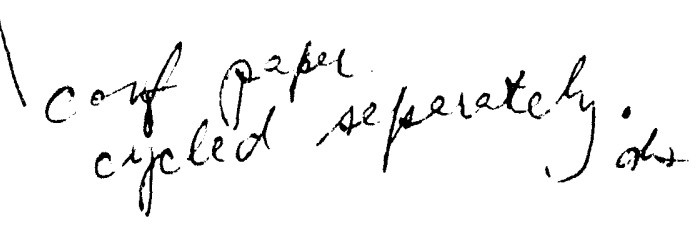


Section 2

EXTRACTION MEASUREIENTS

\subsection{Introduction}

$\mathrm{KrF}$ lasers must be as efficient as possible for these devices to be viable candidates as ICF drivers. Early computer model predictions indicated that the laser efficiency could be improved by minimizing all losses and increasing the gain by increasing the e-beam deposition rate. Both of these effects tend to maximize the gain-to-loss ratio $\left(g_{0} / a\right)$, thereby helping the laser efficiency.

Hence, the goal of this part of the program was to demonstrate high intrinsic efficiency and to determine, as accurately as possible, the parameters needed to calculate this efficiency. This latter goal was critical because generally the weakest aspect of determining the laser efficiency is estimating the input e-beam deposition.

Estimating the e-beam input power deposition is nontrivial because not only must parameters, such as the gas volumes, be accurately known, but also because the reduction of the data must be performed carefully to ensure that all required correction factors are properly included. Hence, part of the work performed during this task included experimental efforts to measure critical parameters needed during the data reduction process. For example, the nonuniformity of the e-beam deposition in the active volume was measured.

A versatile Faraday cup design was also developed and tested. This diagnostic tool proved to be very useful during the course of the program and has already been used extensively in the follow-on work that STI is currently performing. Details of the Faraday cup design and testing are given in Appendix A. Where appropriate, data taken with the cup is presented in this section. 
The rest of this section describes the extraction experiment, the determination of the input e-beam deposition, and discusses the results.

\subsection{Description of Experiment}

The experiment used the Tahoma e-beam pumped laser facility that has been used extensively in the past to perform experiments on $\mathrm{KrF}, \mathrm{XeCl}, \mathrm{XeF}$, and $\mathrm{KrCl}$. It is an exceptionally well-characterized device, which makes it an ideal tool for examining the various $\mathrm{KrF}$ laser issues facing the LANL model development program.

The laser consists of a $375 \mathrm{kV}$ Marx bank that energies a carbon felt, cold cathode and produces an e-beam whose dimensions are $10 \times 70 \mathrm{~cm}$. Total e-beam current at the foil [0.13 mm (0.005 inch) aluminized Kapton] is $\simeq 16$ $\mathrm{kA}$ with a peak current density of $32 \mathrm{~A} / \mathrm{cm}^{2}$. An external magnetic field of 2000 gauss is used to guide the e-beam into the laser gas chamber. The ebeam pulse width is variable between $0.1-1 \mu \mathrm{s}$, but for most of the rork performed during this program the pulse length was fixed at 450 ns.

Several improvements were performed on the laser during the program to reduce resonator losses and increase the e-beam deposition. Two sources of optical loss that can be controlled are absorption and reflection from resonator windows, and absorption by fluorine in portions of the gas volume outside the active volume. The best way to entirely remove losses by the windows is to eliminate them altogether and use the resonator mirrors to contain the gas. If the mirrors are also positioned inside the gas chamber very close to the ends of the e-beam, this will minimize the amount of unexcited gas in the resonator volume, thereby reducing the amount of absorption caused by fluorint.

Although re-entrant mirrors help reduce optical losses, there is the drawback that the mirror lifetime is shorten significantly due to halogen attack of the dielectric coating. Hence, during the experiments, the 
mirrors were inspected frequently and replaced at the first signs of degradation.

Beyond using re-entrant mirrors, there is no other easy way to further reduce nonsaturable losses. Therefore, the next improvements to the laser were to increase the gain by increasing the e-beam deposition. The transmission of the anode screen in the e-beam diode chamber was increased from $58 \%$ to $70 \%$ by using a screen with wider openings. A lead lined e-beam stop was installed in the laser chamber to back scatter electrons into the active volume. This is shown schematically in Figure 2-1. The active volume, whose dimensions are $3.8 \mathrm{~cm}$ diameter by $70 \mathrm{~cm}(0.8$ liters), is located near the back wall of the beam stop. Measurements indicate that the beam stop increases the deposition in the active volume by $\simeq 20 \%$.

The beam stop also serves the important function of defining the volume of laser gas excited by the e-beam. Knowing this is critical because it is needed during the e-beam deposition calculation (see Section 2.3) .

These improvements, as well as operating at a higher magnetic field strength of $2 \mathrm{kG}$, resulted in increasing the e-beam deposition to 285 $\mathrm{kW} / \mathrm{cm}^{3}$ (volume averaged over the diameter of the active volume). In the center of the active volume, where the gain and absorption were probed, the deposition is $\simeq 17 \%$ higher $\left(330 \mathrm{~kW} / \mathrm{cm}^{3}\right)$ due to nonuniformities in the e-beam deposition (see Section 2.3).

With the reentrant mirrors and higher e-beam deposition, the extraction experiments consisted primarily of measuring the laser output energy as a function of the gas mixture and output coupling fraction (i.e. output mirror reflectivity). Two gas mixtures were studied. One we shall refer to as "Ar-diluent" consisted of $\mathrm{F}_{2} / \mathrm{Kr} / \mathrm{Ar}=2.8 / 145 / 856$ Torr $(14.5 \%$ $\mathrm{Kr}$ ); and the other we shall call "Kr-diluent" consisted of $\mathrm{F}_{2} / \mathrm{Kr}=3.5 / 662$ Torr. 


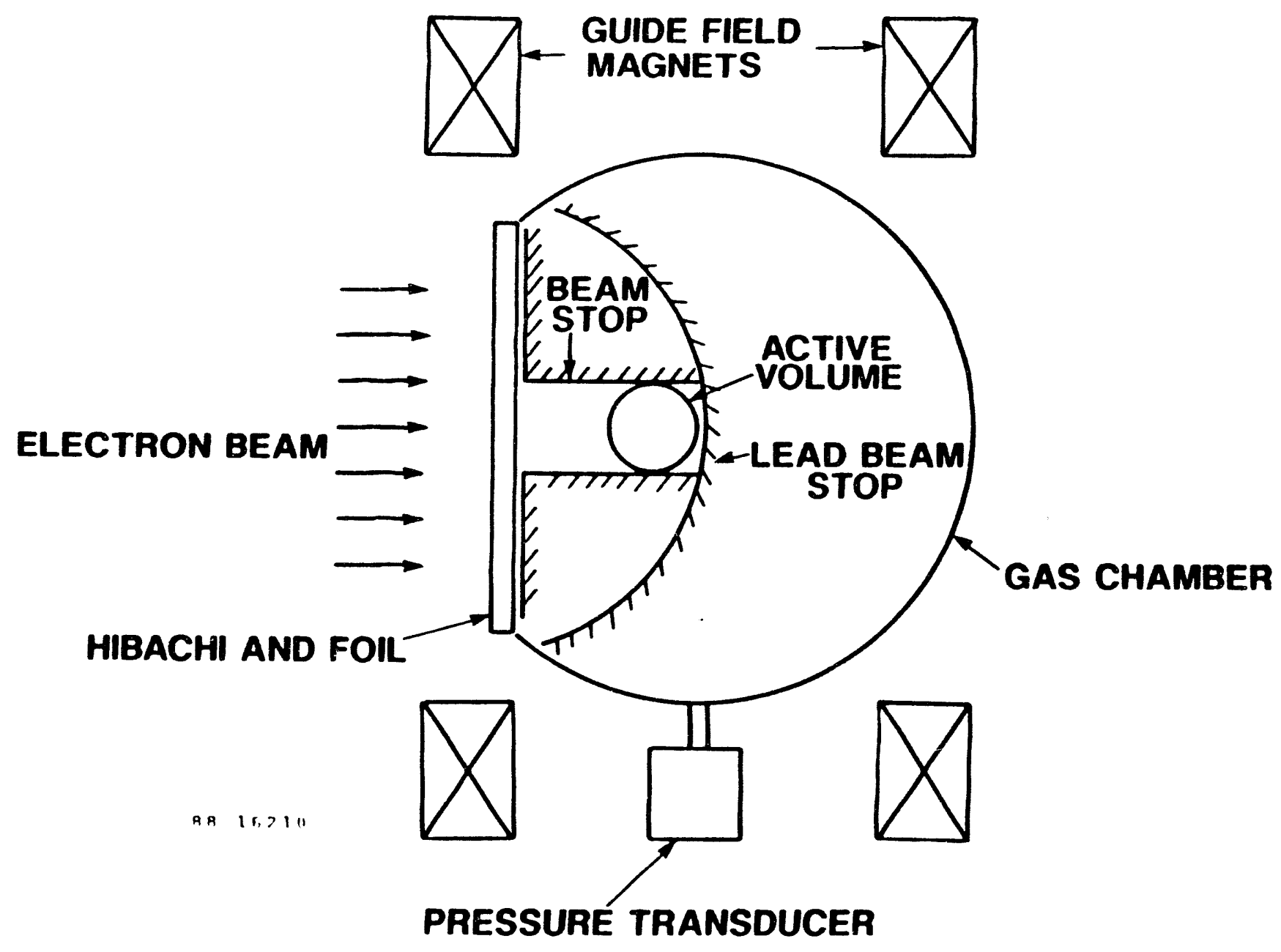

Figure 2-1. Cross-section of Tahoma Laser Chamber. 


\subsection{Determination of B-Bean Deposition}

There are several different methods for measuring the e-beam power deposition into the laser gas. The primary method used during these experiments is the common pressure jump technique, where the transient increase in gas pressure inside the laser chamber caused by heating of the gas by the e-beam is measured using a pressure transducer. All methods require either correction factors and/or the need to interpolate the raw data in order to give the e-beam deposition. Part of the reason for choosing the piessure jump technique over other methods is because: 1) it gives a direct indication of the amount of energy deposited into the laser gas without the need to interpolate the data or adjust for different electron stopping powers; 2) the correction factors for it can be determined with reasonable accuracy; and 3 ) it enables real time measurement of the e-beam deposition during the actual experiments. As an independent check of the deposition determined from the pressure jump data, the Faraday cup together with the LANL Monte Carlo deposition code were used to predict the deposition rate. This is discussed later in this section.

A capacitive manometer (MKS Baratron Type 122A) is used as the pressure transducer. Its position relative to the laser active volume was shown earlier in Figure 2-1. Note that the transducer senses the total volume-integrated pressure of the laser chamber including volumes that are excited by the e-beam and volumes that are outside this excitation region. Consequently, the pressure rise measured by the transducer caused by e-beam energy deposited into the gas volume between the e-beam foil and beam stop (see Figure 2-1) will be diluted by the large volume of gas surrounding the beam stop. This dilution factor can be accurately determined by measuring the relative volumes inside the laser chamber.

Besides measuring the e-beam pumped and unpumped volumes during the experiment, the active volume relative to the pumped volume was also measured (see Figure 2-1). These volume correction factors are used during the e-beam deposition calculation to convert the pressure rise measured by 
the transducer to the equivalent pressure rise occurring inside the laser active volume.

The time response of the pressure transducer is crucial. It must be fast enough to accurately track the pressure rise. (Including equilibration time, this is estimated to be several milliseconds.) During the program, the UKS Baratron response was cross-checked by a fast (2 ms response time) differential transducer (Datametrics Model 570 sensor). This differential transducer had a limited operating range of 10 Torr pressure differential; whereas, the typical pressure rise during the experiments is $\simeq 60$ Torr. Hence, the cross-check could only be performed at reduced total gas pressure. Nonetheless, it was found that the time response of the MKS Baratron unit was being limited by its electronics rather than its intrinsic mechanical characteristics. Modifying the electronics improved the response time to $\leq 50 \mathrm{~ms}$; however, this is still slower than desired. Fortunately, the two transducers agree with each when the MKS Baratron response is extrapolated to the same point in time when the differential transducer reaches its peak ( $~ 20 \mathrm{~ms}$ ). This is how the pressure rise from the MKS Baratron was read during the experiments. [A new, faster ( 1 ms response time) Baratron unit (Type 317H) has been recently installed on the laser to augment the limited capability of the slower transducer.]

Another important diagnostic whose calibration was checked is the laser power meter (Scientech 38-2UV5) used to measure the laser output energy. Typically, these meters are calibrated under cw conditions. Since the experiments are always under pulsed conditions, it was important to verify that the $c$ calibration was still valid. A calibration system was built to pulse the heater coil in the meter sensing head used to calibrate the device. Pulses as short as $8 \mathrm{~ms}$ were tested and it was found that the cw calibration was still accurate for these pulsed conditions. (Although the laser pulse length is < $1 \mu \mathrm{s}$, we believe with regard to pulsed response characteristics, the power meter behavior will be essentially the same for millisecond or microsecond pulses.) 
Other correction factors to the pressure jump relate to the intrinsic properties of the laser medium and e-beam. The first is to account for $\mathrm{KrF}^{*}$ fluorescence emission or energy loss. When excited by the e-beam, the gas emits photons that do not result in heating up the gas and, therefore, are not detected by the pressure transducer. The amount of fluorescence emission depends upon whether the laser is lasing or nonlasing. (Indeed, when calculating the total energy deposited into the gas during lasing conditions, one must be careful to include the energy represented by the laser output itself.) Until recently, computer model predictions for the fluorescence emission had to be relied upon. The model at STI predicted for our experimental conditions fluorescence energy loss of $\simeq 8 \%$ and $\simeq 18 \%$ during lasing and nonlasing conditions, respectively. McCown, et al.(2-1) recently measured the fluorescence energy loss under nonlasing conditions in $\mathrm{KrF}$ to be $18 \%$. This is in very good agreement with the model value we have been assuming.

The next and final factor concerns any nonuniformity of the e-beam deposition within the active volume. If the e-beam deposition is nonuniform this will not be manifested in the pressure jump data because the pressure transducer integrates over all volumes. To measure the e-beam deposition profile within the active volume, a fiber optic sensing system was developed to collect the $\mathrm{KrF}^{*}$ emission as a function of position. (The assumption being that the $\mathrm{KrF}^{*}$ emission profile directly follows the e-beam deposition profile.) This is shown schematically in Figure 2-2. A special holder positions the ends of eight UV optical fibers in a row facing the laser gas. The fiber ends are actually recessed inside the holder to control the numerical aperture of the fibers. To limit the viewing volume of each fiber and to ensure that light from a viewed volume is not collected by more than one fiber, an opaque light barrier is positioned $\simeq 4$ $\mathrm{cm}$ away from the fiber holder block. The fiber holder is designed to be either positioned horizontally (as shown in Figure 2-2) to measure the ebeam deposition profile away from the foil, or vertically to measure the deposition profile across the e-beam face (the same plane as shown in Figure 2-1). This latter profile turns out to be the most important with regard to deposition nonuniformities. 


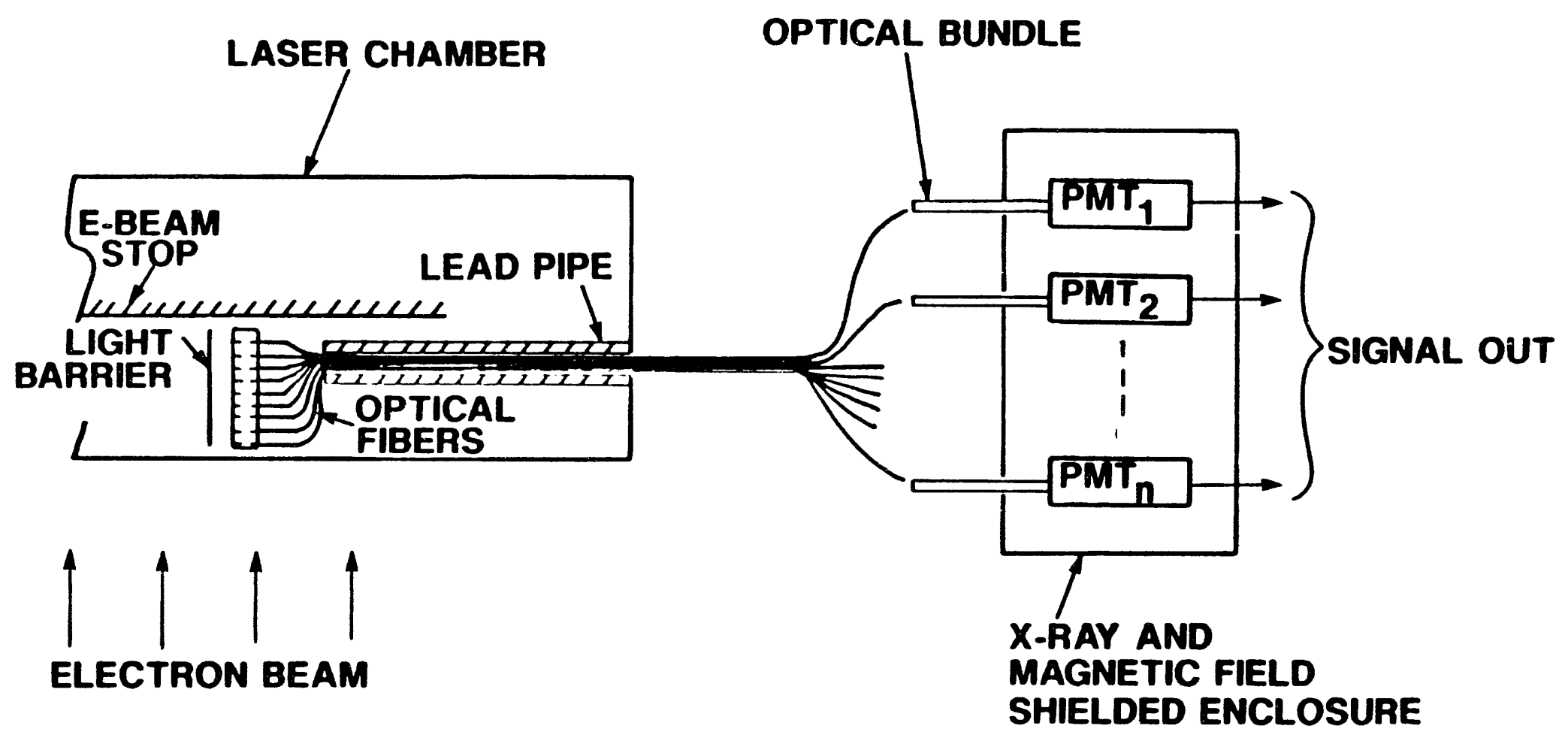

ค ค 1 1 : : :

Figure 2-2. Schematic of E-Beam Deposition Profile Measurement System.

Not shown are lenses and a narromband filter used between the fibers and the PMT system to isolate only the $248 \mathrm{~nm}$ light. 
To protect the fibers from radiation and e-beam damage, the fibers are fed from the holder block through a lead pipe and then outside of the laser chamber. Each fiber is fed into separate photomultiplier tubes housed in an $x$-ray and magnetic field shielded enclosure. Not shown in Figure 2-2 are lenses and a $248 \mathrm{~nm}$ narrowband filter positioned between the fiber ends and the photomultiplier inputs to eliminate extraneous light signals.

The e-beam deposition profile results using the fiber optic sensing system are given in Figure 2-3. Figures 2-3(a) and (b) show the horizontal and vertical profiles, respectively, with the error bars on the data points representing the fluctuations over a series of e-beam shots. The solid curves are the results from the LANL Monte Carlo deposition code for the conditions of our experiment. ${ }^{(2-2)}$ The code results have been arbitrarily scaled to $f$ it the data in order to compare profile shapes. Therefore, one should not interpret the results depicted in Figure 2-3 as indicating agreement in absolute value between the data and model. Later, it will be shown that on a volume-averaged basis, the pressure jump and Monte Carlo results agree with each other.

Both the data and model indicate that the deposition in the horizontal direction is fairly flat, especially in the region occupied by the active volume [range $1.6 \mathrm{~cm}$ to $5.4 \mathrm{~cm}$ on the abscissa in Figure 2-3(a)]. However, in the vertical direction [Figure 2-3(b)], the deposition is quite nonuniform. Again, the data and model agree quite well with each other in overall shape. The net effect of the nonuniformity in the vertical direction is that the deposition is slightly higher in the active volume than if the deposition were completely uniform.

Understanding how these various correction factors are incorporated into the calculation of the laser efficiency can be explained by first referring to Figure 2-4, which shows a simplified cross-sectional view of the part of the laser chamber volume pumped by the e-beam (see Figure 2-1). On the left side of the rectangular region (labeled Region 2) is the e-beam foil; top and bottom are the walls of the beam stop; and on the right side 


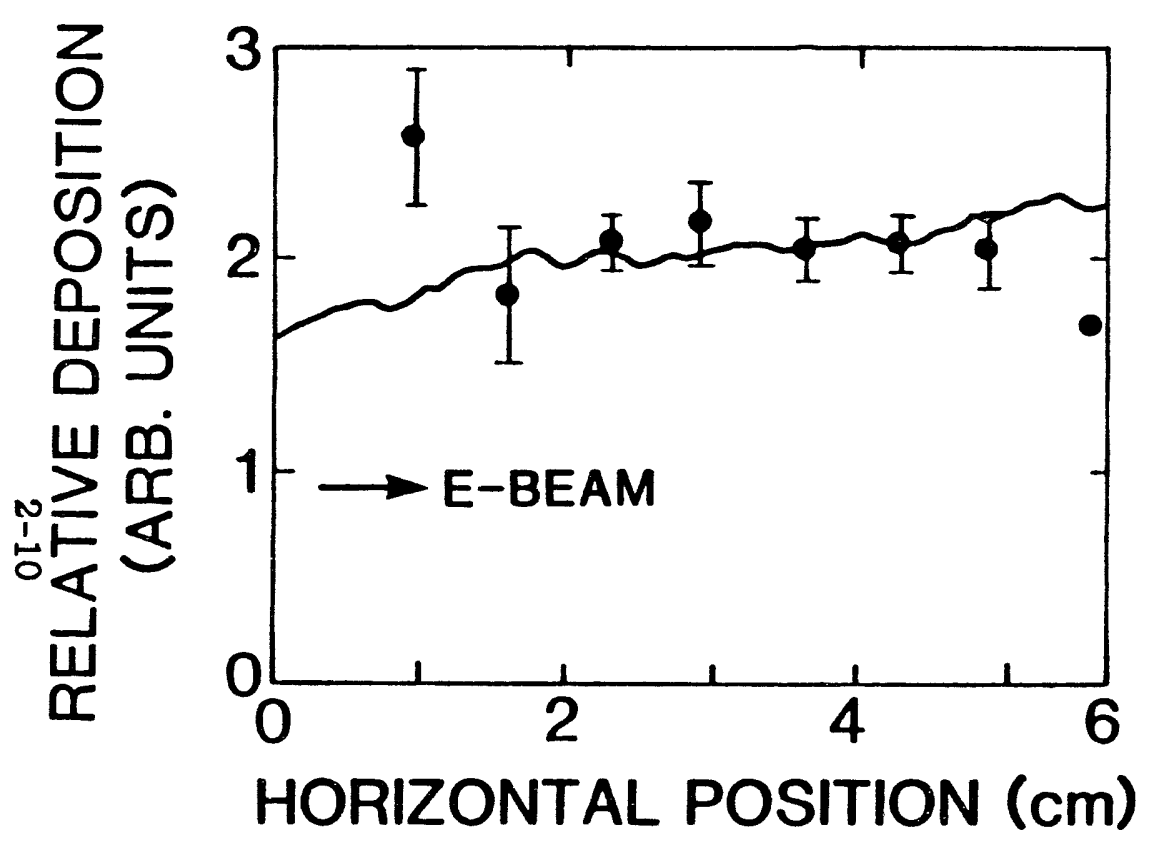

(a)

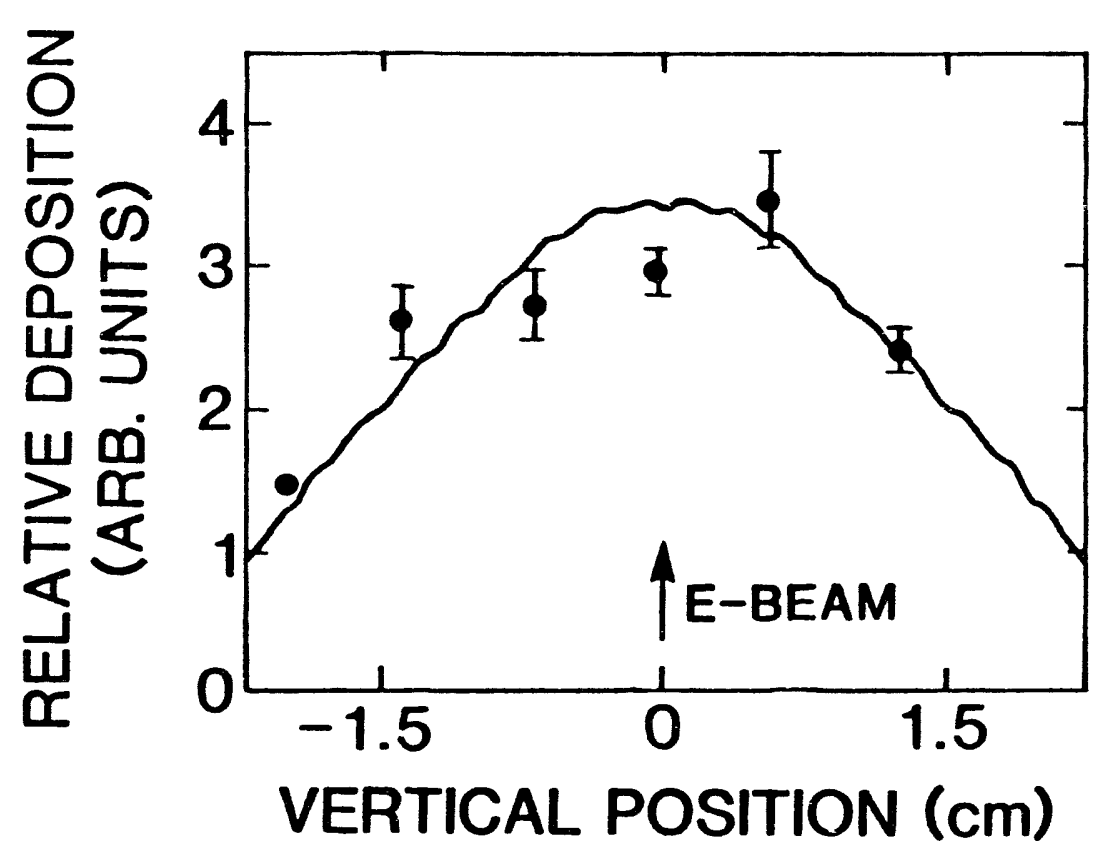

(b)

Figure 2-3. E-Beam Deposition Profile Results. The data points are from the fiber optic measurements; the curve is from the LANL Monte Carlo E-beam deposition code. (a) Deposition profile in horizontal direction away from the e-beam foil. (b) Deposition profile in vertical direction along the e-beam foil. 


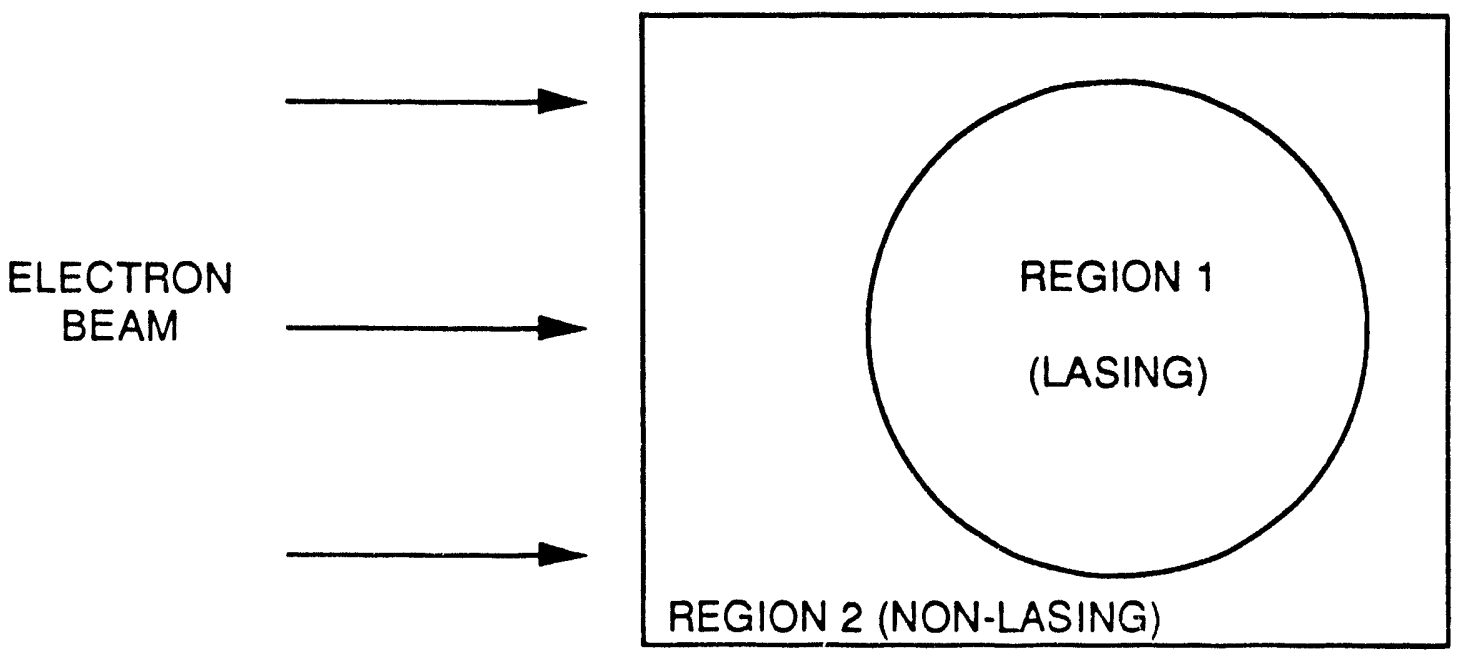

8917767

Figure 2-4. Schematic Representation of the E-beam Pumped Regions Within the Laser Chamber With the Beam Stop in Place. 
is the beam stop back wall (in reality it is concave inward). The active volume (labeled Region 1) is outlined as a circular region representing the diameter of the laser windows. As mentioned earlier, the pressure rise, $\Delta \mathrm{P}$, measured during the experiment is due to energy deposited into both regions.

Both Regions 1 and 2 emit fluorescence photons, but because only Region 1 is lasing, the amount of fluorescence energy loss from both regions are not identical. Using the fluorescence energy loss values mentioned earlier, the energy deposited in both regions, $E_{\text {dep' }}$ is given by,

$$
E_{\text {dep }}=2.77 \Delta P[J / \text { Torr }]+0.08 E_{\text {dep } 1}+0.18 E_{\text {dep } 2}+E_{\text {lase }} \text {, }
$$

where $E_{\text {dep1 }}$ and $E_{\text {dep2 }}$ are the energy deposited in Regions 1 and 2, respectively, and $E_{1 \text { ase }}$ is the laser output energy. The derivation of the factor, 2.77 $\triangle \mathrm{P}$, is explained in detail in Appendix B, which analyzes the effects of nonuniform temperature distributions in the laser chamber during the pressure rise measurements. Equation 1 shows that the amount of fluorescence energy loss depends on the deposition energy in the region of interest.

The e-beam pumps both Regions 1 and 2, and because of e-beam nonuniformities the deposition is not the same in both regions. However, before incorporating the effects of nonuniformity, let us assume that the deposition is uniform, i.e. $E_{\text {dep1 }}=E_{\text {dep2 }}$, then Eqn. 1 reduces to,

$$
E_{\text {dep }}=\frac{2.77 \Delta P+E_{\text {lase }}}{1-\frac{1}{V_{\text {tot }}}\left[0.08 v_{1}+0.18 V_{2}\right]} \text {, }
$$

where $V_{1}$ and $V_{2}$ are the volumes of Regions 1 and 2 , respectively, and $V_{\text {tot }}=$ $v_{1}+v_{2}$. The deposition in only Region 1 is then,

$$
E_{\text {dep } 1}=E_{\text {dep }} \frac{V_{1}}{V_{\text {tot }}}
$$


The laser efficiency, assuming uniform e-beam deposition in both regions, is given by,

$$
\eta=\frac{E_{\text {lase }}}{E_{\text {dep }}}=\frac{2.02 E_{\text {lase }}}{2.77 \Delta P+E_{\text {lase }}},
$$

in which the measured values of the volumes of each region have been used $\left(V_{1} / V_{\text {tot }}=0.43\right.$ and $\left.V_{2} / V_{\text {tot }}=0.57\right)$. An average efficiency is obtained by averaging the measured efficiencies from multiple shots.

As mentioned before, Equation 4 is valid only for uniform pumping and the correction for nonuniformities must be included. Using the e-beam deposition profile results from the fiber optics measurement, the actual volume averaged deposition in the two regions relative to the uniform deposition case is,

$$
\begin{aligned}
& \mathrm{E}_{\text {dep } 1}=1.22 \mathrm{E}_{\text {dep }}\left[\mathrm{V}_{1} / \mathrm{V}_{\text {tot }}\right] \\
& \mathrm{E}_{\text {dep2 }}=0.84 \mathrm{E}_{\text {dep }}\left[\mathrm{V}_{2} / \mathrm{V}_{\text {tot }}\right] .
\end{aligned}
$$

Equation 2 then becomes,

$$
E_{\text {dep }}=\frac{2.77 \Delta P+E_{\text {lase }}}{1-0.128},
$$

and Eqn. 3 changes to,

$$
\mathrm{E}=1.22 \mathrm{E}_{\text {dep }} \frac{\mathrm{V}_{1}}{\mathrm{~V}_{\text {tot }}} \text {, }
$$

so that the laser efficiency, including the effects of nonuniform e-beam deposition, is now given by,

$$
\eta=\frac{1.83 \mathrm{E}_{\text {lase }}}{2.77 \Delta \mathrm{P}+\mathrm{E}_{\text {lase }}}
$$


Comparing Eqns. 4 and 7, we see that the effect of the nonuniform deposition is to reduce the laser efficiency from the uniform deposition case by $\simeq 10 \%$.

\subsection{Experimental Results}

Using the reentrant mirrors, lead beam stop, and $285 \mathrm{~kW} / \mathrm{cm}^{3}$ volume averaged e-beam deposition rate, the Tahoma laser output at $248 \mathrm{~nm}$ was measured as a function of krypton concentration. The intrinsic laser efficiency, as calculated from the data using Eqn. 7, is plotted in Figure 2-5. The optical resonator consisted of a $\operatorname{Max} R$ (10 m radius of curvature) rear reflector and a flat output coupler. Optimum output reflectivity for the conditions of this experiment is $10 \% \mathrm{R}$.

Table 2-1 lists the specific gas mixture compositions for the data given in Figure 2-5. Besides varying the krypton concentration, the fluorine concentration was also adjusted to improve the laser performance (especially for the $\mathrm{Kr}$-diluent case), and the total pressures of the gas mixtures were selected to achieve the same power deposition for each case.

Representative photodiode traces of the laser output and $\mathrm{KrF}^{*}$ sidelight fluorescence are given in Figure 2-6. Figures 2-6(a) and (b) are for the Ardiluent and $\mathrm{Kr}$-diluent cases, respectively. The sidelight is monitored by using a photodiode attached to an observation port underneath the laser chamber. A metal tube joins the port hole inside the laser chamber to a hole in the beam stop in order to permit collection of light from the active volume. This helps reduce the amount of extraneous light outside the active volume being detected by the photodiode, but it does not entirely eliminate it. We estimate that as much as $50 \%$ of the collected light is outside the active volume. Nonetbeless, it is possible to partially observe the $\mathrm{KrF}$ fluorescence suppression occurring during lasing. This is illustrated in Figure 2-7, which shows the sidelight fluorescence under nonlasing conditions [Figure 2-7(a)] and lasing conditions [Figure 2-7(b)] for the Ar-diluent case with $20 \%$ R output reflector. (In order to enable lasing and nonlasing operation, these data were obtained without the reentrant mirrors.) Note, 


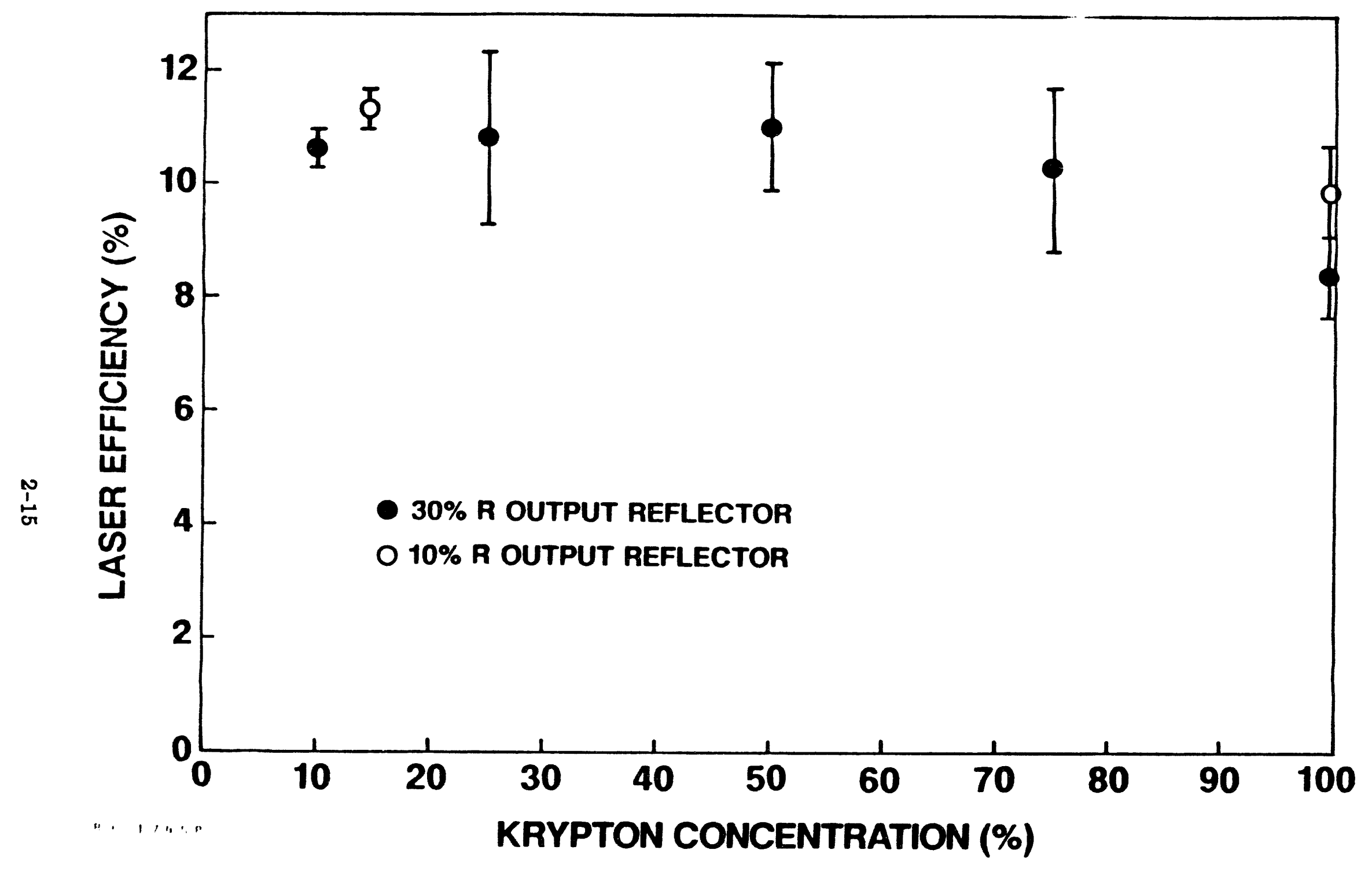

Figure 2-5. Intrinsic Laser Efficiency as a Function of the Initial Krypton Concentration. 


\section{Table 2-1}

Sumary of Gas Mixture Compositions During Bxtraction Measurements

$$
\text { (All values in Torr) }
$$

Krypton

Concentration

$\% \mathrm{Kr}$

10

14.5

25

50

75

98.6

$0 / 661 / 3.5$ $10 \% \mathrm{R}$

$\mathrm{Ar} / \mathrm{Kr} / \mathrm{F}_{2}$

$858 / 142 / 2.8$
Output Reflector

$30 \%$ B

$\underline{\mathrm{Ar}} / \mathrm{Kr} / \mathrm{F}_{2}$

$801 / 100 / 2.7$

$690 / 231 / 3.0$

$405 / 410 / 3.5$

$116 / 614 / 3.5$

$0 / 661 / 3.5$ 


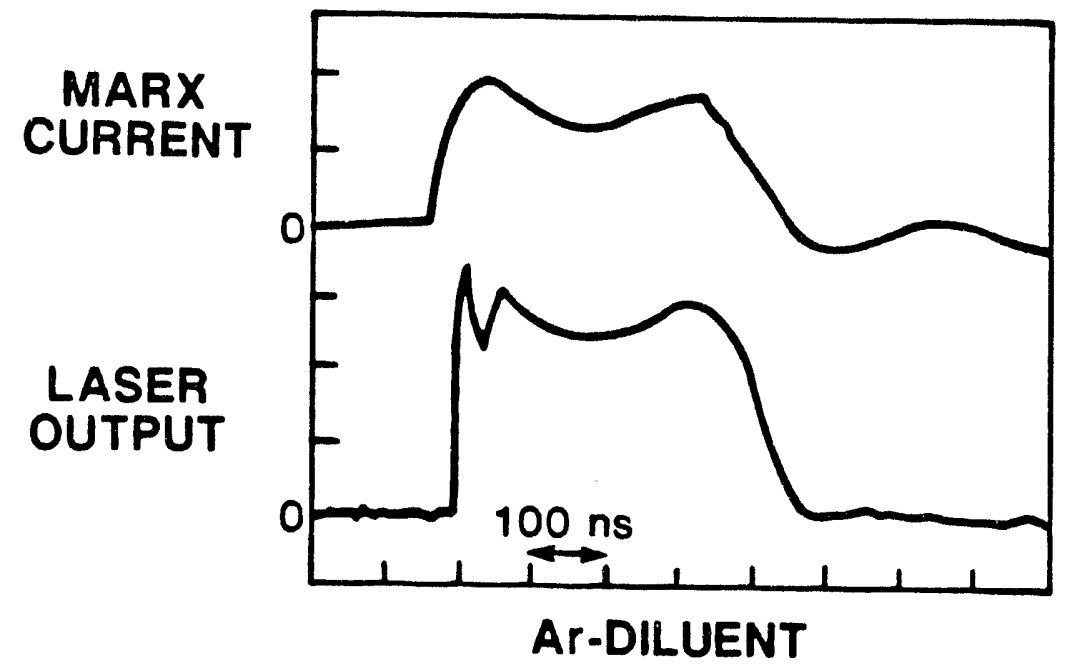

(a)

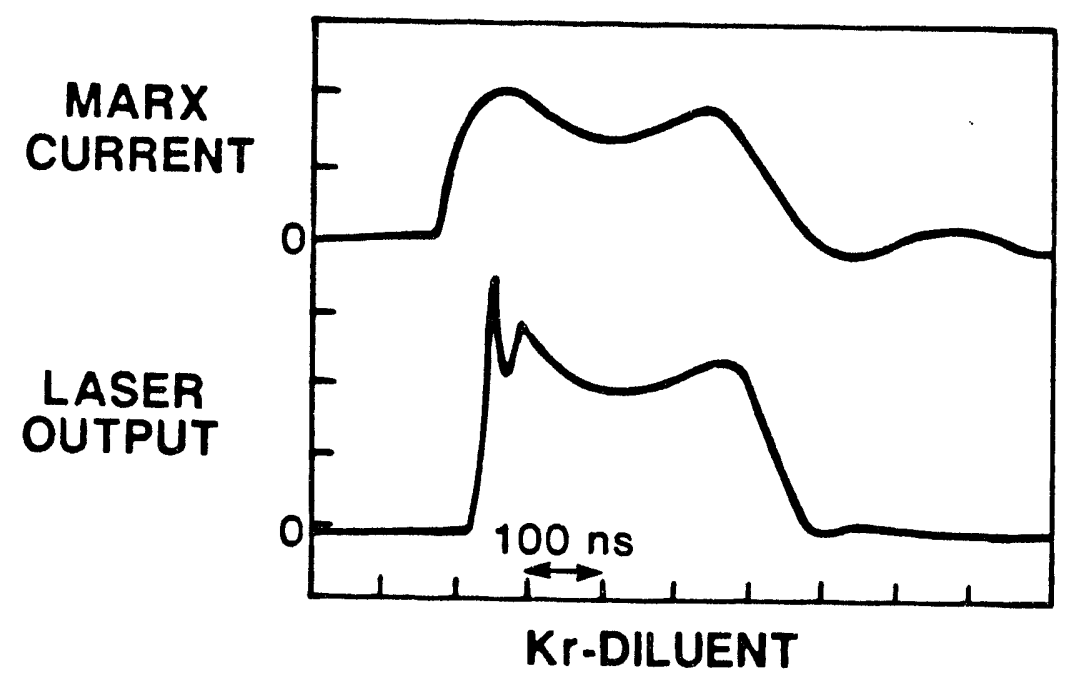

(b)

Figure 2-6. Marx Current and Laser Output Waveforms. (a) For the Ardiluent case. (b) For the $\mathrm{Kr}$-diluent case. 


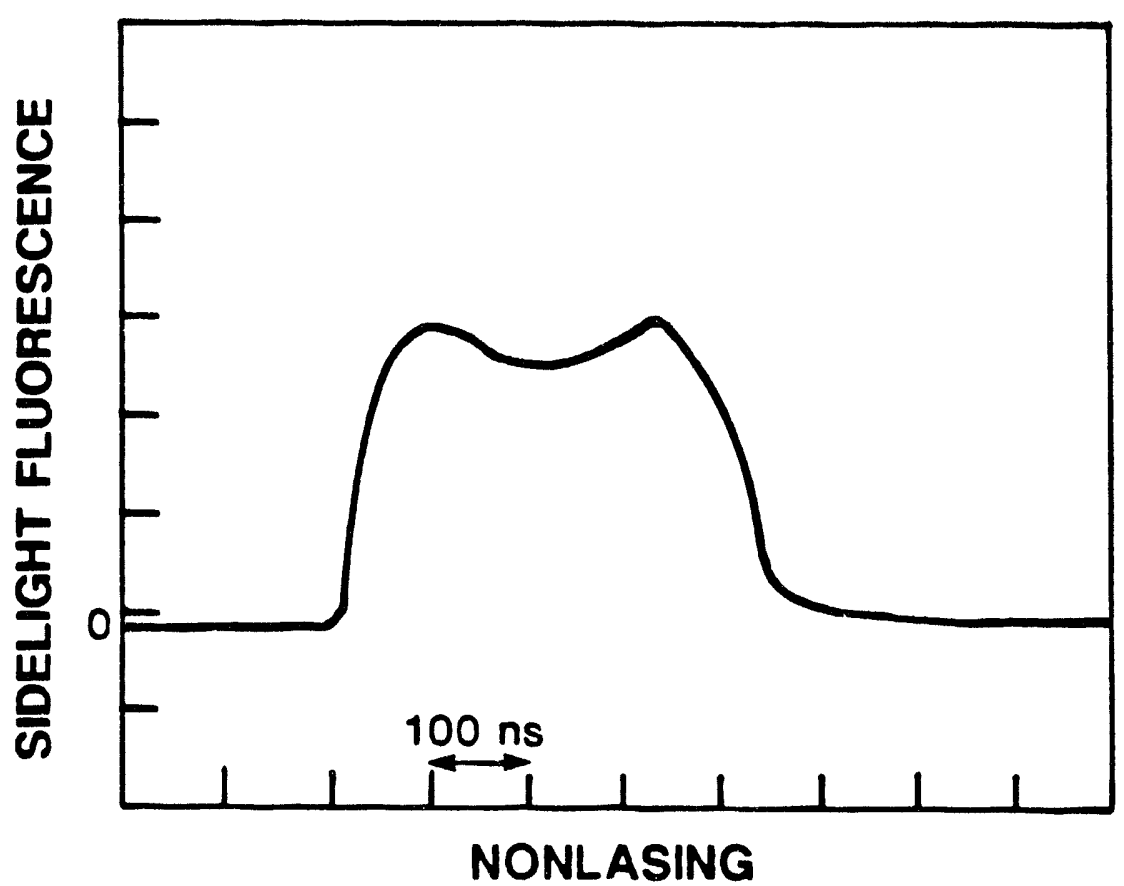

(a)

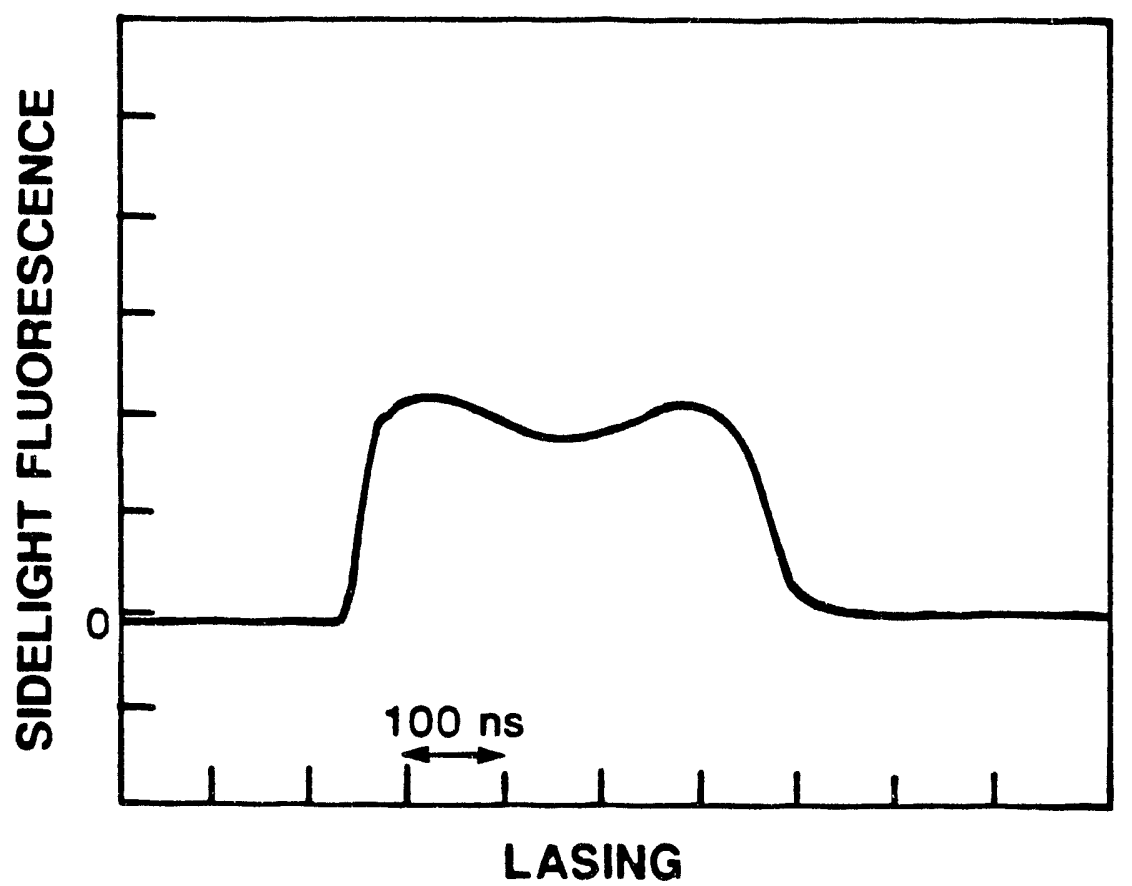

(b)

Figure 2-7. $\mathrm{KrF}^{*}$ Sidelight Fluorescence Waveforms. (a) Under nonlasing conditions. (b) Under lasing conditions. Note, approximately $50 \%$ of the light being detected is from outside the active volume. 
that even with extraneous light being collected, a suppression in fluorescence signal of roughly $25 \%$ is observed.

\subsection{Discussion}

The results given ir. Figure 2-5 indicate a maximum intrinsic laser efficiency of $11.3 \%$ at $14.5 \% \mathrm{Kr}$ concentration. However, the laser efficiency is fairly insensitive to the krypton concentration when between 10 to 70 percent krypton, so that the efficiencies at these concentrations could very well be comparable to the value at $14.5 \% \mathrm{Kr}$. For the $\mathrm{Kr}$-diluent case, there is a definite tendency for poorer performance, even with the optimum $10 \% \mathrm{R}$ output reflector. Indeed, closer examination of the laser output waveforms previously given in Figure 2-6, show that the laser output for the Kr-diluent mixture is slightly reduced from that for the Ar-diluent case. Why there is such a rather sudden decrease in performance between the $70 \% \mathrm{Kr}$ and $\mathrm{Kr}$-diluent cases is not understood. Additional discussion regarding the data are presented in Section 3.

\subsection{References}

2-1. A.W. YcCown, T.P. Turner, R.A. Tennant, and B.J. Krohn, "Determination of Fluorescence Energy Loss From an Electron-Beam Pumped KrF Laser," Conference on Lasers and Electro-Optics, 1988 Technical Digest Series, Vol. 11 (Optical Society of America, Washington D.C., 1989) p. 340.

2-2 Courtesy of B.J. Krohn, LANL. 
Section 3

GAIN AND TRANSIENT ABSORPTION MEASUREYRNTS

\subsection{Introduction}

The gain and absorption of the laser medium are obviously two very important parameters that directly affect the laser efficiency. Any model of the laser must be able to accurately predict these quantities. Although gain and absorption measurements have been performed in the past, the objective of this work was to provide this data as part of a comprehensive data package to be used to help verify the LANL model. Hence, the conditions of the laser system were kept intentionally the same as the extraction measurements except for some minor differences that should not affect the results.

Indeed, as discussed in Section 3.5, a self-consistency check of several fundamental quantities using the gain, absorption, and laser efficiency data (see Section 2.4), demonstrate that the data are selfconsistent to one another. This is reassuring since, as will be discussed, preliminary comparisons with the LANL model have already revealed some significant disagreements.

\subsection{Description of Experiment}

A schematic of the gain and absorption measurement system is shown in Figure 3-1. A pulsed Nd:YAG laser tripled to $355 \mathrm{~nm}$ pumps a dye laser whose output is doubled into the UV using a BBO crystal cut for $250 \mathrm{~nm}$. This variable wavelength output is split into a reference beam, which goes directly into a photodiode, and a probe beam, which passes twice through the Tahoma laser active region and, after an optical delay, eventually into the same photodiode. Employing a single photodiode eliminates the need to calibrate multiple detectors. The optical delay is long enough to allow viewing both reference and probe signals without temporal overlap. The 


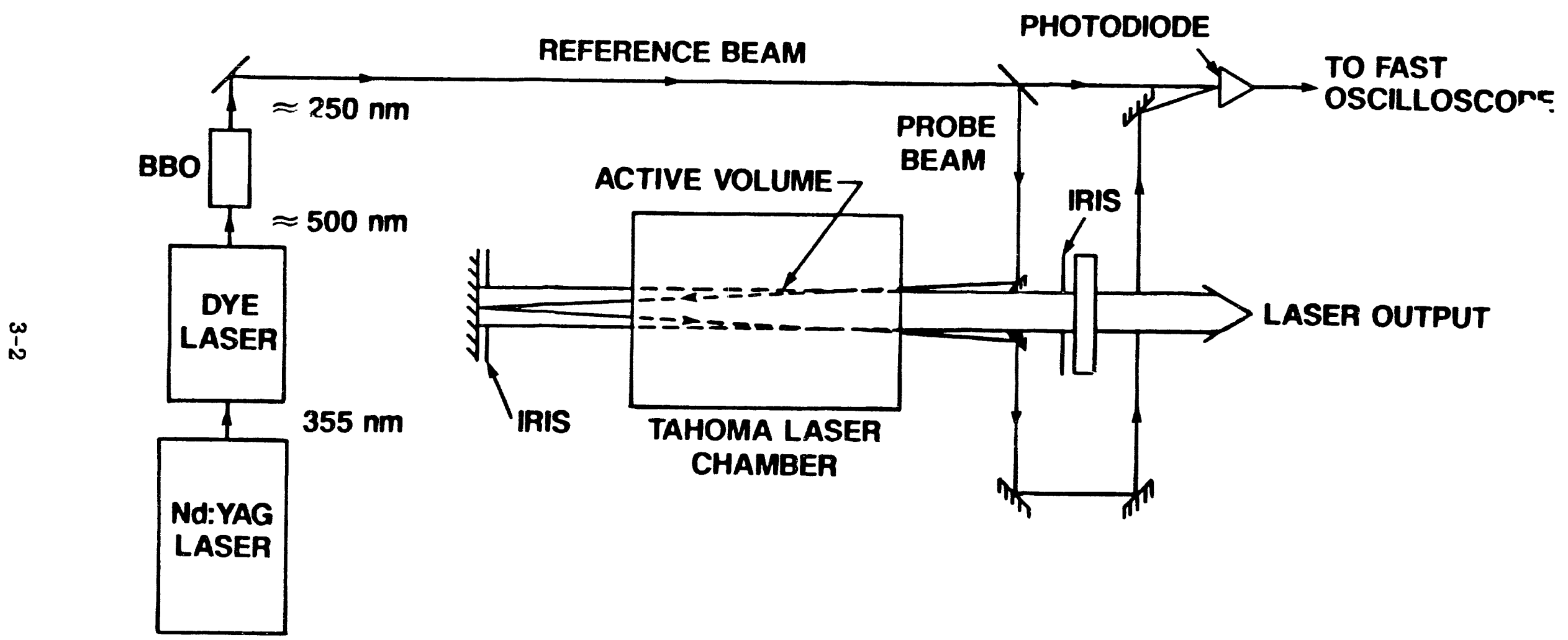

Figure 3-1. Schematic of the Gain and Transient Absorption Measurement System. 
probe is inserted into and extracted from the oscillator cavity without interfering with the intracavity flux by using small turning prisms. Intracavity irises control the output laser beam diameter to prevent coupling any significant laser radiation into the prisms and the photodiode. Note that the rear high reflector mirror of the laser cavity also serves as the middle mirror for the double pass of the probe beam. Although a convenient method for enabling the double pass of the probe beam, this feature also tends to complicate the analysis of the absorption data because of amplified spontaneous emission (ASE) effects during nonlasing conditions. This will be discussed later. The intracavity flux is varied by simply changing the output coupler.

When measuring gain, the probe beam is tuned to $248 \mathrm{~nm}$. Of course, the absorption right at $248 \mathrm{~nm}$ cannot be measured by probing at $248 \mathrm{~nm}$; instead, absorption measurements are performed at wavelengths on either side of the $248 \mathrm{~nm}$ line and the absorption at $248 \mathrm{~nm}$ is inferred by performing a linear interpolation between the two off-line values. The gain band of $\mathrm{KrF}$ is wide enough that one must probe at least $\simeq 8 \mathrm{~nm}$ on either side of the line center to avoid any appreciable gain contributions. The wavelengths chosen for the off-line absorption measurements are 240.0 and $257.3 \mathrm{~nm}$.

\subsection{Analysis Technique}

Figure 3-2 shows schematically the geometry during the gain and absorption measurements. As mentioned, the probe beam passes twice through the laser chamber, which consists of both pumped and unpumped regions, and windows (the re-entrant mirror system was not used during these measurements). $I_{i n}$ and $I_{\text {out }}$ designate the input and output probe intensities, respectively. Before $I_{\text {in }}$ enters the laser chamber it is sampled using a beam splitter (see Figure 3-1). This reference signal serves to monitor fluctuations in the probe beam power and any long term window degradation. After an optical delay, $I_{\text {out }}$ is sent into the photodiode. As will be explained, it is necessary to measure the probe 

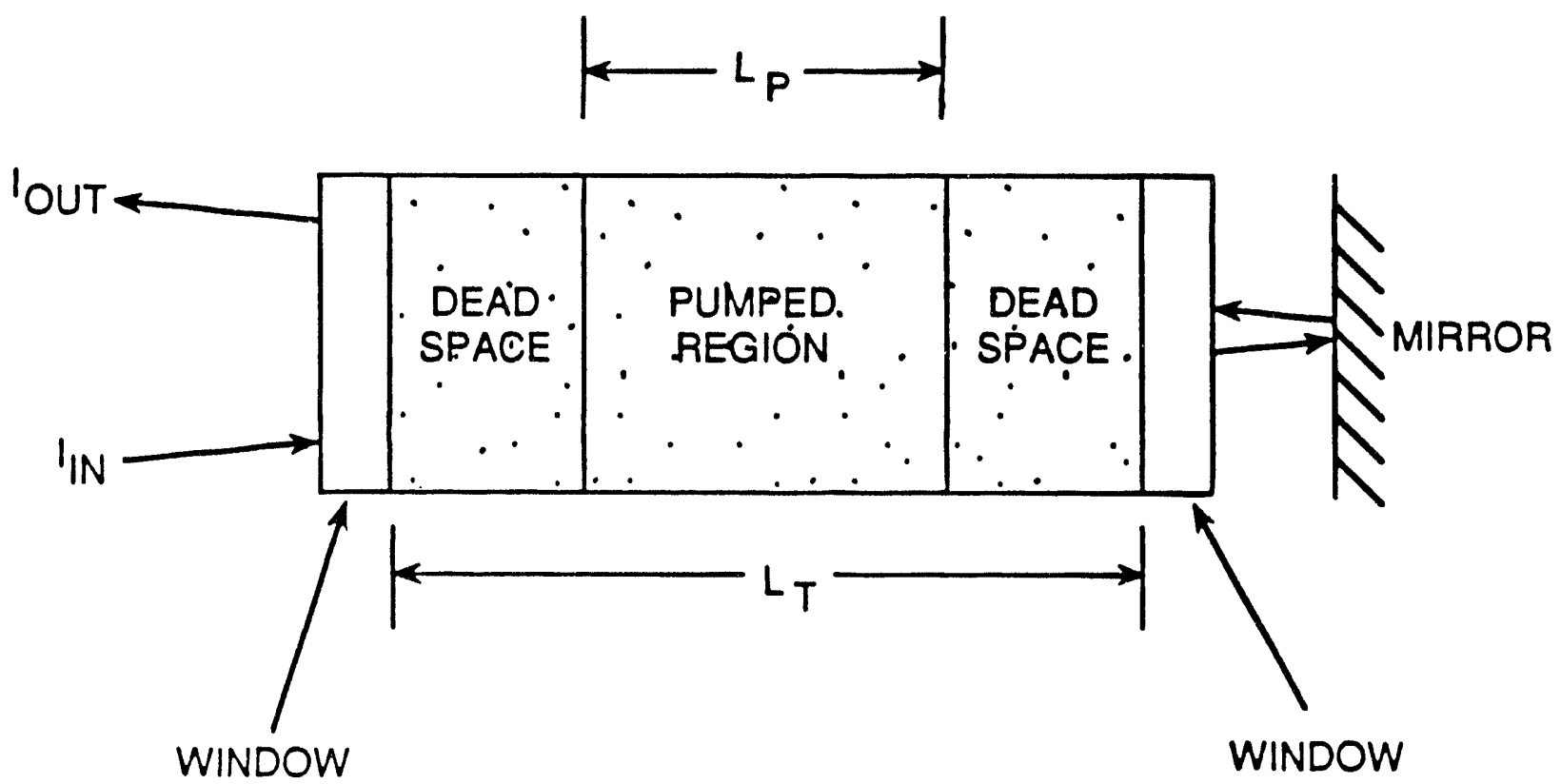

$$
\begin{aligned}
I_{I N} & =\text { INPUT PROBE INTENSITY } \\
\text { IOUT } & =\text { OUTPUT PROBE INTENSITY } \\
L_{T} & =\text { TOTAL LENGTH OF GAS REGION } \\
L_{P} & =\text { LENGTH OF PUMPED REGION }
\end{aligned}
$$

8917136

Figure 3-2. Schematic Representation of the Probe Beam Propagation Conditions During the Measurements. 
intensities during both pumped and unpumped conditions, and the intensity data itself must be compared as ratios with the reference signal.

To derive from the intensity measurements the net gain and absorption in only the pumping region, three sets of data must be collected. These are :

$$
\begin{aligned}
& {\left[\frac{I_{\text {out }}}{I_{\text {in }}}\right]_{\text {with } \mathrm{KrF} \text { mix before shot }} \equiv I_{\text {cal }}} \\
& {\left[\frac{I_{\text {out }}}{I_{\text {in }}}\right]_{\text {with } \mathrm{KrF} \text { mix during shot }} \equiv r_{\text {shot }}} \\
& {\left[\frac{I_{\text {out }}}{I_{\text {in }}}\right]_{\text {in vacuum }} \equiv r_{\text {vac }}}
\end{aligned}
$$

Typically, $r_{\text {vac }}$ is determined only once at a given wavelength, while $r_{\text {shot }}$ and $r_{c a l}$ constitute a data set that is obtained for each shot. During unpumped conditions, the transmission factor for a fresh gas mixture is determined at each wavelength by utilizing consecutive $r_{\text {vac }}$ and $r_{c a l}$ measurements. Because fresh mixtures are used for every shot, the gas transmissions for unpumped conditions need only be obtained once. For pumped conditions, however, the situation is different. Window degradation affects $I_{\text {out }}$ as well as the gain and absorption of the excited gas. As a result, to correct for effects extrinsic to the intrinsic properties of the laser mixture being measured, $r_{c a l}$ is determined before each shot.

In terms of these intensity ratio definitions, the gain/absorption dependence is derived for the pumped region as follows. Referring to Figure 3-2, the probe is influenced by three regions in the laser chamber. Losses occur at the windows, mirror, and unpumped regions (dead space), and a combination of gain and loss occurs in the pumped region. The effect each region has on the probe beam can be described independently by first lumping the static losses in the following way: 
$T_{w}=$ Total 2-pass transmission through the windows.

$\begin{aligned} T_{B}= & \text { Total 2-pass transmission through the gas during unpumped } \\ & \text { conditions }\end{aligned}$

$T_{d}=$ Total 2-pass transnission through only the gas-filled dead spaces

$\mathbb{R}=$ Reflectivity of the mirror

The intensity measurements are related to the above quantities in the following manner:

$$
\begin{aligned}
& r_{c a l}=T_{w} T_{g} R \\
& r_{v a c}=T_{w} R \\
& r_{\text {shot }}=T_{w} T_{d} R \exp \left[2(g-a) L_{p}\right],
\end{aligned}
$$

where $g=$ gain and $a=$ loss.

Next, normalizing to the reference signals,

and

$$
\frac{r_{\text {cal }}}{r_{\text {vac }}}=T_{g} \text {, }
$$

$$
\frac{r_{\text {shot }}}{r_{\text {cal }}}=\frac{T_{d}}{T_{g}} e^{2(g-a) L_{p}}
$$

Given that the absorption per unit length of the unpumped gas is constant over the entire length $L_{t}$, then,

$$
T_{g}=e^{-\beta L_{t}} \text { and } T_{d}=e^{-\beta\left(L_{t}-L_{p}\right)}
$$


where $\beta=$ absorption per unit length. It follows that,

so that,

$$
\frac{T_{d}}{T_{g}}=e^{-\beta\left(-L_{p}\right)}=\left[e^{-\beta L_{t}}\right]^{-L_{p} / L_{t}}=T_{g}{ }^{-L_{p} / L_{t}}
$$

$$
\frac{r_{\text {shot }}}{r_{\text {cal }}}=T_{g}^{-L_{p} / L_{t}} e^{2(g-a) L_{p}} .
$$

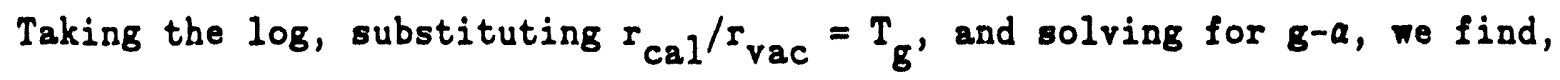

$$
g-a=\frac{1}{2 L_{p}} \ln \left[\frac{r_{\text {shot }}}{r_{\text {cal }}}\right]+\frac{1}{2 L_{t}} \ln \left[\frac{r_{c a l}}{r_{\text {vac }}}\right] .
$$

Equation 3-1 is used when reducing the data for both the on-line gain and off-line absorption measurements.

As mentioned earlier, the feedback from the bigh reflector during nonlasing conditions aggravates the ASE loading within the cavity. This potentially impacts the absorption measurements because during nonlasing there may still be an appreciable amount of intracavity flux present due to ASE. An ASE code developed at STI ${ }^{(3-1)}$ predicts $520 \mathrm{~kW} / \mathrm{cm}^{2}$ of flux loading based on the gain measurements made by probing on-line center at $248.4 \mathrm{~nm}$. As will be shown, the saturable loss component $a_{s}$ requires $>1 \mathrm{~mW} / \mathrm{cm}^{2}$ to saturate. Consequently, the ASE effects can be considered negligible and can therefore be neglected.

\subsection{Gain and Transient Absorption Results}

Table 3-1 lists the intensity ratio data $\left(r_{\text {shot }} / r_{\text {cal }}\right)$ and their uncertainties for most of the conditions studied. The beam stop is the same one used during the extraction experiments (see Section 2.2). It increases the pumping in the active volume by $\simeq 20 \%$. The intensity ratios are given with and without the beam stop in the laser chamber (high and low deposition, respectively), at three wavelengths, and for the Ar-diluent and 
Table $3-1$
INTENSITY RATIO DATA $\left(\frac{\mathbf{r}_{\text {shot }}}{\mathbf{r}_{\text {cal }}}\right)$

Results without Beam Stop in Laser Chamber

NONLASING

WAVELENGTH $(\mathrm{nm})$

\begin{tabular}{|c|c|c|c|}
\hline Gas Mix ${ }^{(1)}$ & 240.0 & 248.4 & 257.3 \\
\hline $14.5 \% \mathrm{Kr} / \mathrm{F}_{2} / \mathrm{Ar}$ & $0.401 \pm 0.01$ & $2411 \pm 190$ & $0.373 \pm 0.09^{(2)}$ \\
\hline $99.6 \% \mathrm{Kr} / \mathrm{F}_{2}$ & $0.246 \pm 0.05$ & $1382 \pm 120$ & $0.176 \pm 0.04$ \\
\hline
\end{tabular}

$\underset{\infty}{\omega} \quad$ Results with Beam Stop in Laser Chamber

$\begin{array}{llll}14.5 \% \mathrm{Kr} / \mathrm{F}_{2} / \mathrm{Ar} & 0.153 \pm 0.01 & 9722 \pm 1200 & 0.347 \pm 0.01 \\ 99.6 \% \mathrm{Kr} / \mathrm{F}_{2} & 0.317 \pm 0.02 & 1908 \pm 190 & 0.202 \pm 0.02 \\ 100 \% \mathrm{Kr} & & & 0.389 \pm 0.02\end{array}$

(1)

$F_{2}$ Partial Pressure: 2.8 Torr $\quad$ Total Pressure: $\quad\left(\begin{array}{lll}14.5 \% & \mathrm{Kr} r \\ 99.6 \% & \mathrm{Kr}\end{array}\right) \begin{array}{r}1004 \\ 665\end{array}$ Torr

(2) Gas Mixture Contained $10 \% \mathrm{Kr}$ for this Data
LASING (90\%R)

WAVELENGTH (nm)

240.0

248.0

257.3

$0.534 \pm 0.09$

$3.08 \pm 0.21$

$0.484 \pm 0.05(2)$

$0.362 \pm 0.04$

$3.37 \pm 0.27$

$0.323 \pm 0.03$

$0.560 \pm 0.04 \quad 2.57 \pm 0.20 \quad 0.445 \pm 0.02$

$0.400 \pm 0.02$

$2.29 \pm 0.04$

$0.372 \pm 0.02$ 
$\mathrm{Kr}$-diluent gas mixtures during lasing and nonlasing conditions. A $90 \% \mathrm{R}$ reflector is used for the lasing conditions to ensure that the laser is well saturated (recall that the optimum output reflectivity for this laser is $10 \% \mathrm{R}$ ). For comparison purposes, a pure krypton mixture is probed at $257.3 \mathrm{~nm}$ during both pumping conditions.

The static transmission of the gas mixtures are not listed in the table. They are:

$$
\begin{aligned}
& {\left[\mathrm{T}_{\mathrm{g}}\right]_{240.0 \mathrm{~nm}}=0.83} \\
& {\left[\mathrm{~T}_{\mathrm{g}}\right]_{248.4 \mathrm{~nm}}=0.80} \\
& {\left[\mathrm{~T}_{\mathrm{g}}\right]_{257.3 \mathrm{~nm}}=0.74}
\end{aligned}
$$

Uncertainties in these values are less than \pm 0.05 .

Employin Equation 3-1, Table 3-2 shows the reduced data from Table 3-1. All values are in $\% / \mathrm{cm}$. Table 3-3 lists the gain and absorption coefficients at $248.4 \mathrm{~nm}$. The gain coefficients are calculated directly from the on-line measurements. Both the small signal and nonsaturable absorptions are derived by performing a linear interpolation between the off-line measurements. Gain-to-loss ratios are also listed.

The overall trends being displayed by the data can be seen better in Figure 3-3, which plots the gain and absorption as a function of the e-beam deposition. Also plotted are the initial LANL model predictions ${ }^{(3-2)}$ for the Tahoma laser conditions.

The model tends to underpredict both the unloaded (nonlasing) and loaded (lasing) gain. Only a small difference in seen between the Ardiluent and $\mathrm{Kr}$-diluent gain values for both the data and model. Although there is not a large separation between the two different e-beam deposition rates, the gain data does seem to increase at a rate similar to that predicted by the model. 
Table 3-2

GAIN/LOSS VALUBS ${ }^{(2)}$ (\%/cm)

BEAY STOP OUT

Wavelength $(\mathrm{nm})$ :

$14.5 \% \mathrm{Kr} / \mathrm{F}_{2} / \mathrm{Ar}^{(3)}$

99.6\% Kr/P ${ }_{2}^{(3)}$

$100 \% \mathrm{Kr}$

BEAY STOP IN

$\begin{array}{ll}\omega & 14.5 \% \mathrm{Kr} / \mathrm{P}_{2} / \mathrm{Ar} \\ \stackrel{1}{\circ} & (3)\end{array}$

99.6\% Kr/ $/ \mathrm{F}_{2}{ }^{(3)}$

$100 \% \mathrm{Kr}$
NONLASING

240.0
-0.65
$-1.10^{(1)}$

248.4

5.45

257.3

$-0.86^{(4)}$

5.05

$-1.40^{(1)}$

$-0.72$

$\begin{array}{ll}6.44 & -0.91 \\ 5.28 & -1.30 \\ & -0.67\end{array}$

LASTNG (90\%R)

240.0

248.4

257.3

$-0.55$

0.69

$-0.68^{(4)}$

$-0.82$

0.75

$-0.87$

Typical Uncertainties: $\Delta_{\text {gain }}= \pm 0.1 \quad \Delta_{\text {los8 }}= \pm 0.06$

(1) $\Delta_{1088}= \pm 0.15$

(2) Includes correction for unpumped gas regions

(3) $F_{2}$ Partial Pressure : 2.8 Torr Total Pressure: $(14.5 \% \mathrm{Kr}) 1004$ Torr

(4) Gas Mixture Contained $10 \% \mathrm{Kr}$ for this Data

$-0.50$

0.56

$-0.74$

$-0.75$

0.47

$-0.86$ 
Table 3-3

GAIN/LOSS SUTHRT AT $248.4 \mathrm{~mm}(\mathrm{x} / \mathrm{cm})$

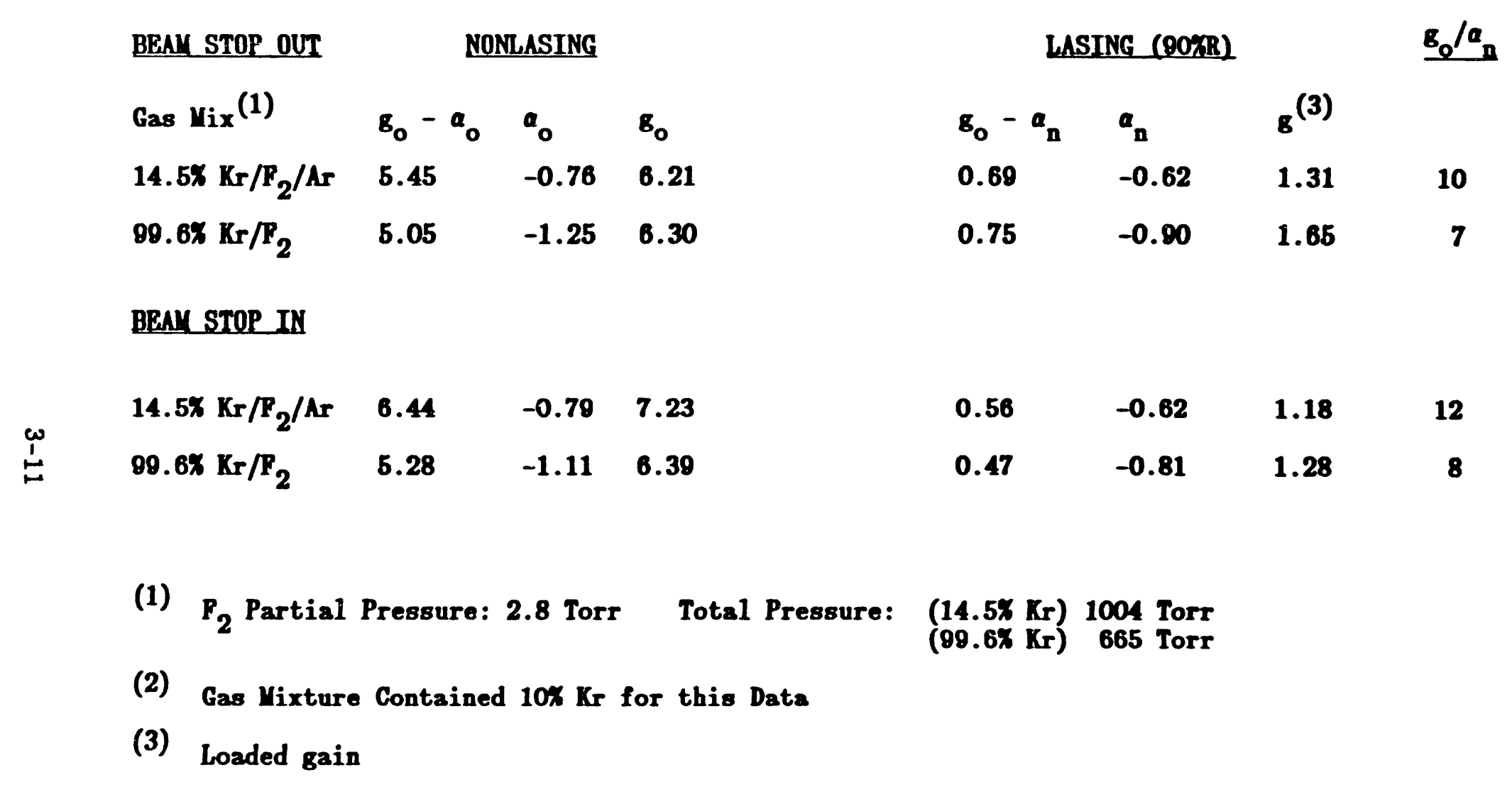



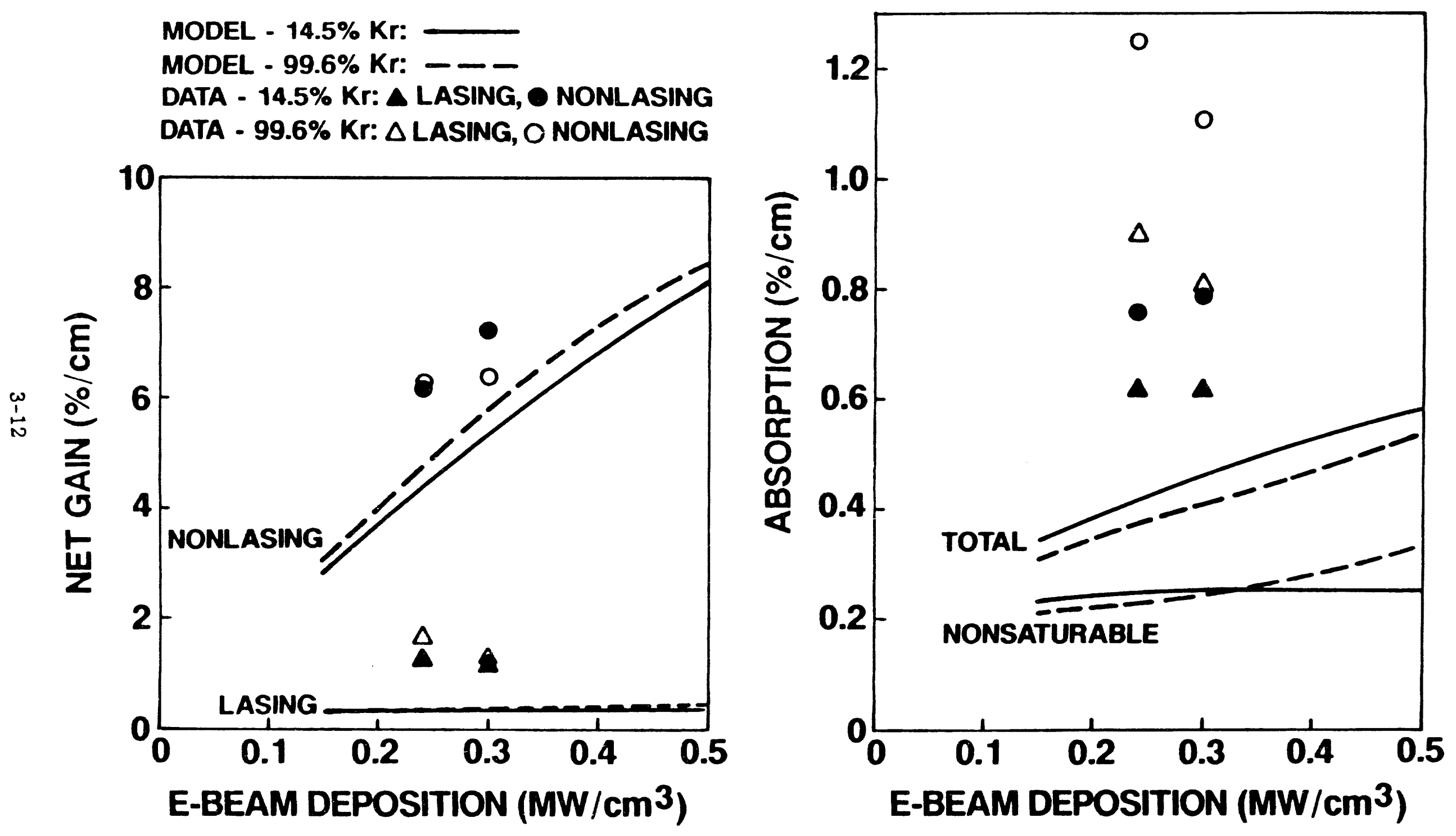

Figure 3-3. Comparison of the Gain and Absorption Data With the LANL Mode1 Predictions. 
The greatest disagreement between the data and model is with the absorption data. The data indicates much higher absorption under both lasing and nonlasing conditions than the model. It appears the nonsaturable component of the absorption (i.e. the lasing data) is a larger fraction of the total absorption than that predicted by the model. Perhaps the most interesting difference between the data and model is that the $\mathrm{Kr}$ diluent data has larger absorption than the Ar-diluent data; whereas, the model predicts just the opposite.

The absorption was also measured as a function of the intracavity flux by varying the output mirror reflectivity. The results for the off-line wavelengths are shown in Figure 3-4. Note that at fluxes greater than approximately $1 \mathrm{~mW} / \mathrm{cm}^{2}$ the absorption values level off to a value representing the nonsaturable absorption component. This implies that the saturation flux for the $\mathrm{KrF}$ laser medium is around $1 \mathrm{~mW} / \mathrm{cm}^{2}$ (a more precise estimate is given in the next section.)

\subsection{Data Analysis}

With the gain, absorption, and intrinsic efficiency data, it is possible to perform simple first-order checks to verify that the data are all self-consistent to one another. First, a power efficiency analysis can be performed by using the relationships,

$$
\begin{aligned}
& \eta_{\text {power }}=\eta_{\text {extraction }} \eta_{\text {formation }} \\
& \eta_{\text {extraction }}=\left[1-\left(g_{0} / a\right)^{-1 / 2}\right]^{2} .
\end{aligned}
$$

where $\eta_{\text {power' }} \eta_{\text {extraction' }}$ and $\eta_{\text {formation }}$ are the power, extraction, and formation efficiencies, respectively. 


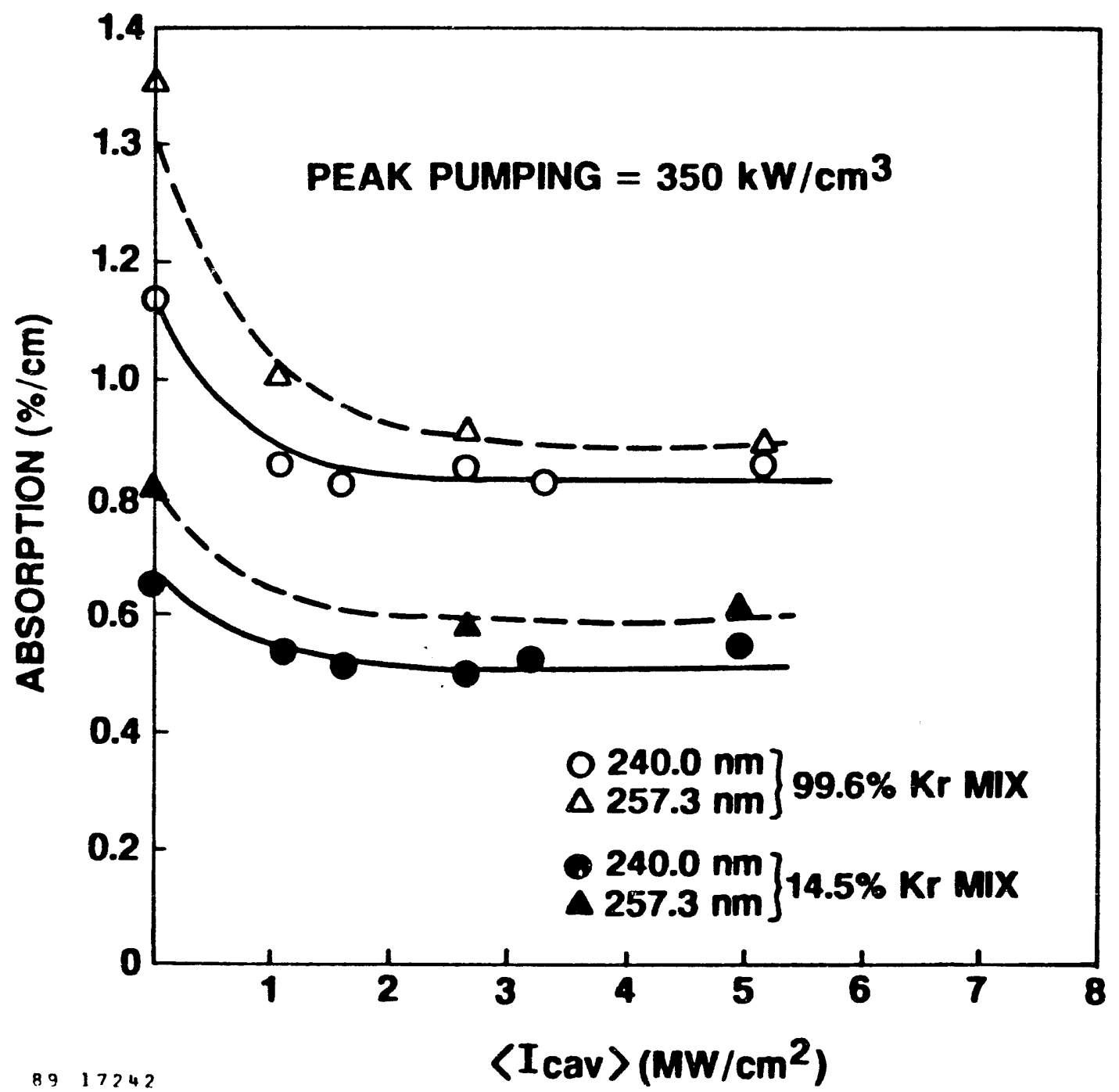

Figure 3-4. Measurements of the Off-Line Absorption as a Function of the Average Intracavity Flux. 
From earlier analysis ${ }^{(3-3)}$ under ideal conditions, we know that the formation efficiency for the $\mathrm{Ar}$ and $\mathrm{Kr}$ diluent mixtures is equal to,

$$
\begin{aligned}
& \eta_{\text {formation }}(A r)=0.24, \\
& \eta_{\text {formation }}(K r)=0.28 .
\end{aligned}
$$

And, from the gain and absorption data (see Table 3-3), the gain-to-loss ratios for the high e-beam pumping case (beam stop in) are,

$$
\begin{aligned}
& \text { For Ar-diluent: } g_{0} / a \simeq 12 \\
& \text { For Kr-diluent: } g_{0} / a \simeq 8 .
\end{aligned}
$$

Substituting these values into Equation 3-3, and using Equation 3-2, we find that the power efficiencies as derived from the gain and absorption data are,

$$
\begin{aligned}
& \text { For Ar-diluent: } \eta_{\text {extraction }}=0.51+\eta_{\text {power }}=12 \%, \\
& \text { For } \mathrm{Kr} \text {-diluent: } \eta_{\text {extraction }}=0.42+\eta_{\text {power }}=12 \% .
\end{aligned}
$$

From the actual laser efficiency measurement results (see Section 2.4), the power efficiencies can be calculated by using the laser output energy and the laser pulse waveform. Since these power efficiency values vary during the pulse duration, the average range of these values are given below,

$$
\begin{aligned}
& \text { For Ar-diluent: } \eta_{\text {power }} \simeq 11-12 \%, \\
& \text { For } \mathrm{Kr} \text {-diluent: } \eta_{\text {power }} \simeq 8-10 \%
\end{aligned}
$$

Hence, the power efficiencies calculated from the gain and absorption data agree well with the power efficiencies derived from the extraction measurements for at least the Ar-diluent mixture. The fact the $\mathrm{Kr}$-diluent power efficiency appears lower than the value calculated from the gain and 
absorption data may be caused by a lower extraction efficiency due to high $\mathrm{Kr}_{2} \mathrm{~F}^{*}$ absorption. Under the conditions of the experiment, there may be incomplete saturation of the $\mathrm{Kr}_{2} \mathrm{~F}^{*}$ molecules leading to both higher $\mathrm{UV}$ absorption and 3 -body quenching of $\mathrm{KrF}$.

Utilizing the experimental data, it is possible to use the modified Rigrod analysis given by Rice, et al. ${ }^{(3-4)}$ to estimate more precisely the saturation flux. Their analysis technique is well suited to our particular experimental configuration. For example, it includes the effects of unpumped gas regions and window losses.

As calculated from the data, the saturation fluxes for the Ar-diluent and $\mathrm{Kr}$-diluent mixtures are $1.1 \pm 0.1 \mathrm{NW} / \mathrm{cm}^{2}$ and $1.4 \pm 0.1 \mathrm{~mW} / \mathrm{cm}^{2}$, respectively. This is very consistent with the saturation behavior seen in Figure 3-4. Unfortunately, the LANL model predicts nearly $2 x$ lower saturation flux. This disagreement is still being investigated along with the differences found during the gain and absorption measurements.

A further check regarding the self-consistency of the data can be conducted using the experimental saturation flux values. The maximum extractable energy from a laser medium is given by $g_{0} I_{\text {sat }}$, where $I_{\text {sat }}$ is the saturation flux. Using the small signal gain values from Table $3-3$ and the experimental $I_{\text {sat }}$ values, we find that,

$$
\begin{aligned}
& \text { For Ar-diluent: } \mathrm{g}_{\mathrm{O}} \mathrm{I}_{\text {sat }}=0.072 \times 1.1=78 \mathrm{~kW} / \mathrm{cm}^{3} \text {, } \\
& \text { For } \mathrm{Kr} \text {-diluent: } \mathrm{g}_{\mathrm{o}} \mathrm{I}_{\text {sat }}=0.064 \times 1.4=90 \mathrm{~kW} / \mathrm{cm}^{3} \text {. }
\end{aligned}
$$

Besides Equation 3-2, formation efficiency can also be defined as $\mathrm{g}_{\mathrm{O}} \mathrm{I}_{\text {sat }} / \mathrm{P}_{\text {in }}$, where $\mathrm{P}_{\text {in }}$ is the input e-beam power deposition. In the center of the active region where the gain was probed, $P_{\text {in }}$ is slightly higher than the volume averaged value and it is equal to $330 \mathrm{~kW} / \mathrm{cm}^{3}$. Hence,

$$
\begin{aligned}
& \text { For Ar-diluent: } \eta_{\text {formation }}=79 / 330=0.24, \\
& \text { For } \mathrm{Kr} \text {-diluent: } \eta_{\text {formation }}=90 / 330=0.27 .
\end{aligned}
$$


These values for the formation efficiency are very consistent with the theoretical values given earlier (Equations 3-4 and 3-5). This also lends support to the hypothesis that the power efficiency for the $\mathrm{Kr}$-diluent mixture is being hurt more by a lower extraction efficiency rather than a lower formation efficiency. Additional analysis must be performed in conjunction with the model to fully understand the implications of the data collected during this program.

\subsection{References}

3-1. D.D. Lowenthal, Spectra Technology, Inc. (private communication).

3-2 D.E. Hanson, Los Alamos National Laboratory, (private communication).

3-3 E.T. Salesky and W.D. Kimura, IEEE J. Quant. Elect. QE-21, 1761 (1985).

3-4 J.K. Rice, G.C. Tisone, and E.L. Patterson, IEEE J. Quant. Elect. QE16, 1315 (1880). 


\title{
Section 4
}

\author{
CONCLUSIONS
}

During the experimental phase of this program, several major experiments were completed. These experiments included extraction measurements to ascertain as accurately as possible the intrinsic laser efficiency under experimental conditions that would maximize the laser performance, and gain and transient absorption measurements under lasing and nonlasing conditions. Within each of these experiments were subtasks needed to help improve the accuracy and thoroughness of the measurements. These subtasks included developing a versatile, well characterized Faraday cup to enable the use of the LANL Yonte Carlo deposition code as a crosscheck of the e-beam deposition; careful calibration of the primary diagnostics and improved understanding of their limitations; development of a more complete analysis of the pressure rise data; and development of a fiber optic $\mathrm{KrF}^{*}$ fluorescence detection system for measuring the e-beam deposition profile.

Intrinsic laser efficiencies for the Ar-diluent $\mathrm{KrF}$ mixture of $11.3 \%$ were obtained using a low optical loss resonator and an e-beam deposition rate of $285 \mathrm{~kW} / \mathrm{cm}^{3}$ (volume averaged). The corresponding power efficiency for this mixture was $12 \%$. Estimated extraction and formation efficiencies as derived from the gain, absorption, and extraction measurements are $51 \%$ and $24 \%$, respectively, for the Ar-diluent mixture.

The $\mathrm{Kr}$-diluent mixture had a lower intrinsic efficiency of $9.8 \%$ and a corresponding power efficiency of $10 \%$. It is more difficult to estimate the extraction and formation efficiencies for this mixture because it appears that neither one were quite optimum during the experiment. Our best estimate, which seems consistent with the rest of the data, is an extraction efficiency of $37 \%$ and a formation efficiency of $27 \%$. The lower than expected extraction efficiency may be due to $\mathrm{Kr}_{2} \mathrm{~F}^{*}$ absorption because this molecule may not be well saturated out under the conditions of the 
experiment. Creation of $\mathrm{Kr}_{2} \mathrm{~F}^{*}$ will also tend to reduce the $\mathrm{KrF}^{*}$ formation efficiency.

The data collected during this program has provided a very good start towards reaching the goal of providing the LANL modeling program with a comprehensive database for validation of their codes. Additional data will be added to this database during a follow-on program that was started immediately after the end of this one. Work is currently being performed for this follow-on program and already interesting data have been obtained. Included in this follow-on program are fluorine depletion (burn-up) measurements under lasing and nonlasing conditions, sidelight fluorescence suppression measurements, additional pressure rise related experiments, and time-dependent rare gas excited state density measurements. The rork performed during this past program and to be conducted during the follow-on program will create one of the most comprehensive databases available to date for a known operating condition. 
Appendix $A$

DEVELOPYENT AND CHARACTERIZATION OF A VERSATIIB FARADAY CUP

\section{A.1 Introduction}

Although Faraday cups are a standard, common diagnostic tool for ebeam sources and their technology has been well developed, it is still rather surprising after searching the open literature that there does not seem to exist a comprehensive set of data characterizing a particular design. Indeed, there does not appear to be a single Faraday cup design that can be considered an industry standard.

Since current density data is one of the key parameters related to the e-beam deposition, which directly affects the laser efficiency measurement, it is critical that the diagnostic used to determined the current density be well understood and characterized. With careful design, it should be also possible to operate the cup in a gaseous environment and, therefore, obtain current density information as a function of position away from the foil.

The goals for our Faraday cup development were several fold. First, it was to develop a Faraday cup design that is inherently versatile and easily modifiable in order to fully test and characterize the cup's performance. The second was to keep the design reasonably simple and yet still be able to perform all the functions desired of it. Third, we wanted a design that would permit operation within a gaseous environment as well as a vacuum. And, lastly, the goal of our development strategy included exploring various design requirements in a step-by-step understandable manner that eliminated the "black art" aspects of cup design. Our hope is that such a thorough characterization of the Faraday cup would allow one to develop a standardized design.

Since e-beam source characteristics can vary over a very wide range in current density, e-beam energy, and temporal characteristics, it was 
decided to design our cup for e-beam sources typical of excimer lasers. For these devices, typical electron energies are $250 \mathrm{keV}$ to $1 \mathrm{MeV}$, and typical current densities range from several to $100 \mathrm{~A} / \mathrm{cm}^{2}$.

The remainder of this appendix describes the Faraday cup design and the tests performed to characterize and understand its performance and limitations. The actual application of this cup for work related to the program is described elsewhere in this final report.

\section{A.2 Description of Faraday Cup Design}

When developing a design for a Faraday cup, the primary concerns are generally driven by the e-beam parameters, specifically the magnitude of the current density and the current risetime. When the former quantity is approximately $>1 \mathrm{kA} / \mathrm{cm}^{2}$ this adds complications to the measurement that make the design of the cup more difficult. Some of these complications include gas desorption due to collector plate heating and reverse currents due to induced EMF's. Since the current densities for the intended application of our cup are an order of magnitude lower than $1 \mathrm{kA} / \mathrm{cm}^{2}$, these issues become much less important.

The current risetime affects the desired time response of the cup. For subnanosecond requirements, impedance matching issues and low inductance and capacitance needs complicate the cup design. For $>1$ ns response times, these design issues become much less severe. Fortunately, for typical e-beam pumped excimer lasers, a few nanosecond time response is more than adequate. This is the time response regime our cup was designed for.

Secondary design concerns involve specific application needs. With regard to the physical size of the cup, a large area cup permits significant portions of the e-beam to be sampled while still allowing smaller portions to be examined by simply aperturing the input to the cup. However, due to space constraints within the laser chamber, it may be undesirable to have a large cup. Large, of course, is a relative term. A 
$10 \mathrm{~cm} \times 10 \mathrm{~cm}$ cup sensing area is large compared to a $10 \mathrm{~cm}$ high e-beam, but it is small compared to a $2 \mathrm{~m}$ high e-beam. The cup developed during this program has a maximum e-beam sensing area of $7 \mathrm{~cm} \times 7 \mathrm{~cm}$, which provides a good working size for medium to large ( $\$ 1 \mathrm{~m})$ size cathodes.

Another design consideration is versatility. This is particularly important for our cup because of the need to understand and quantify various possible operating conditions. It is also important because the optimum design that satisfied the goals for the device performance (e.g. operation in a gas) could only be verified by testing various design configurations. A key strategy for making the cup versatile is using modular components that can be easily changed.

With these constraints in mind, Figure $A-1$ is a diagram of the Faraday cup. Figure $\mathrm{A}-1(\mathrm{a})$ is a front view showing the full $7 \mathrm{~cm} \times 7 \mathrm{~cm}$ aperture. (The dashed lines represent the corrugated collector plate underneath the Kapton foil covering the cup opening.) Figure $A-1$ (b) is a cross sectional view depicting the interior components of the cup. The electrons enter from the left through the optional Kapton foil (for vacuum operation the foil is not necessary). This foil is $0.025 \mathrm{~mm}(0.001$ inch) thick and, as will be shown later, it causes minimal attenuation of the e-bead.

Next, the electrons are absorbed by the collector plate [shown as a corrugated carbon plate in Figure $\mathrm{A}-1(\mathrm{~b})]$. This collector plate bolts onto a plate to which one end of the resistor bank is soldered to. The other end of the resistor bank is soldered to the outside end plate of the cup. (The outside walls of the cup consist of individual side plates bolted together to make up the rectangular box.) $A$ wire from the back of the collector plate feeds through the resistor bank to the coax connector at the back of the cup where the signal output is obtained.

Not shown in Figure $A-1$ are the seals and the gas connections on the side of the cup that allow it to be evacuated and/or filled with up to 200 Torr of a gas (either the laser chamber gas or a different gas). The cover plate depicted in Figure A-1 controls the sensing area of the cup. Areas smaller than $7 \mathrm{~cm} \times 7 \mathrm{~cm}$ can be easily obtained by using different cover 


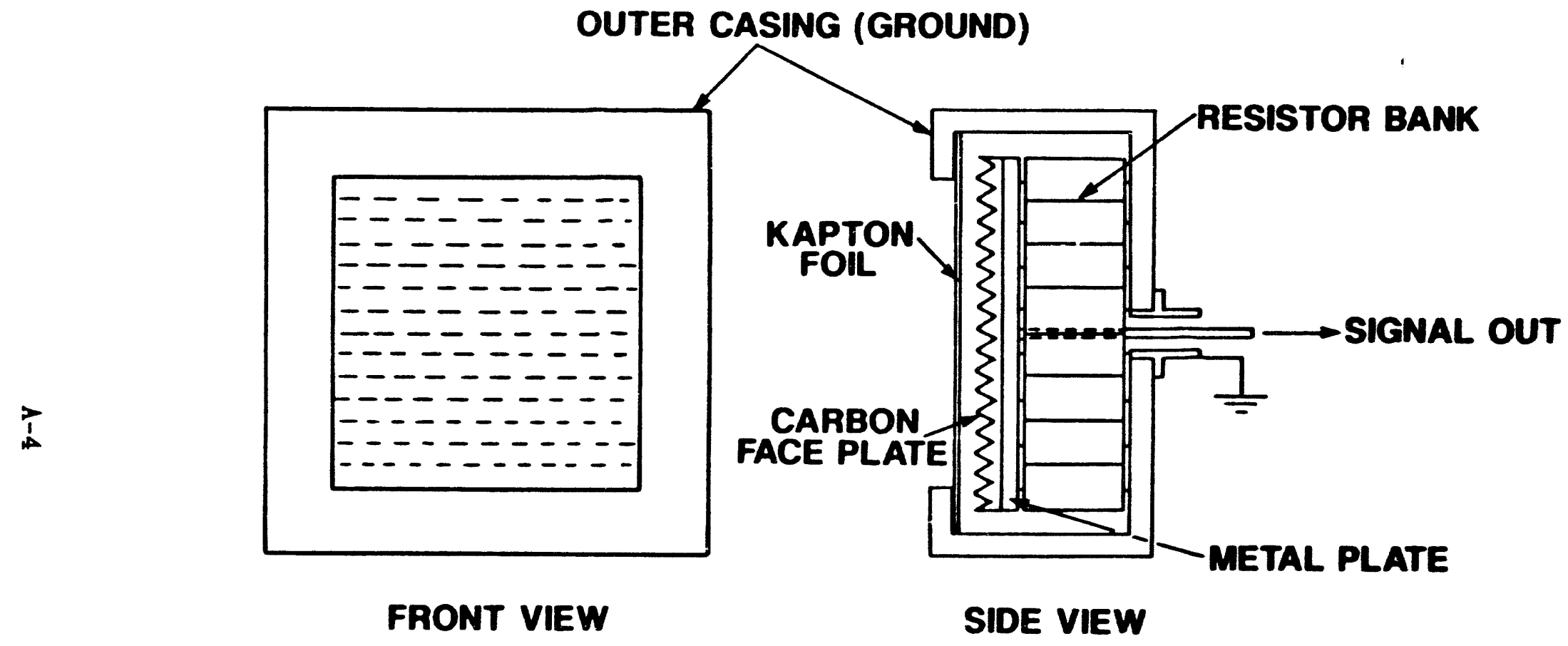

$88 \quad 16211$

(a)

(b)

Figure A-1. Schematic Diagram of the STI Faraday Cup. 
plates with different aperture sizes and locations within the $7 \mathrm{~cm} \times 7 \mathrm{~cm}$ sensing area.

Details of the corrugated collector plate dimensions are given in Figure $1-2$. The $60^{\circ}$ angle of the corrugation is designed to cause any electrons that are reflected off the collector surface to be continually channeled into the groove with each subsequent reflection. As will be shown in Section A.3, although this may help collect reflected electrons, the improvement to the overall output signal for lon $\mathrm{Z}$ (atomic mass) collector materials is negligible.

Figur 1-3 shows the equivalent circuit schematic for the Faraday cup when connected to an oscilloscope. The resistance $R_{s}$ of the resistor bank, which consists of $86,2 \mathrm{~W}, 10 \mathrm{n}$ resistors in parallel, has a measured total resistance of $0.120 \pm 0.002 n$. The capacitance and inductance values are estimated based on the internal geometry of the cup. Pearson probe comparisons performed simultaneous with the Faraday cup (see next section) indicate that the inductance $L_{g}$ and resistance $R_{g}$ are negligible.

Several different types of face plate materials were tested on the cup. These were corrugated carbon and aluminum, and flat beryllium, aluminum, and lead.

\section{A.3 Characterization Tests}

The Faraday cup was tested on the e-beam pumped excimer laser facility called Tahoma. This facility is the same one used during all the experiments; its description was given earlier in Section 2.2. Figure $\mathbf{A - 4}$ shows approximately where the cup is positioned inside the Tahoma laser chamber. The cup is too large to $f$ it inside while the e-beam stop is in place; therefore, all measurements with the cup are taken without the beam stop in place.

To examine the $\mathrm{Z}$-dependence of the collector plate material on measured e-beam current, various plates were tested with the cup operating 


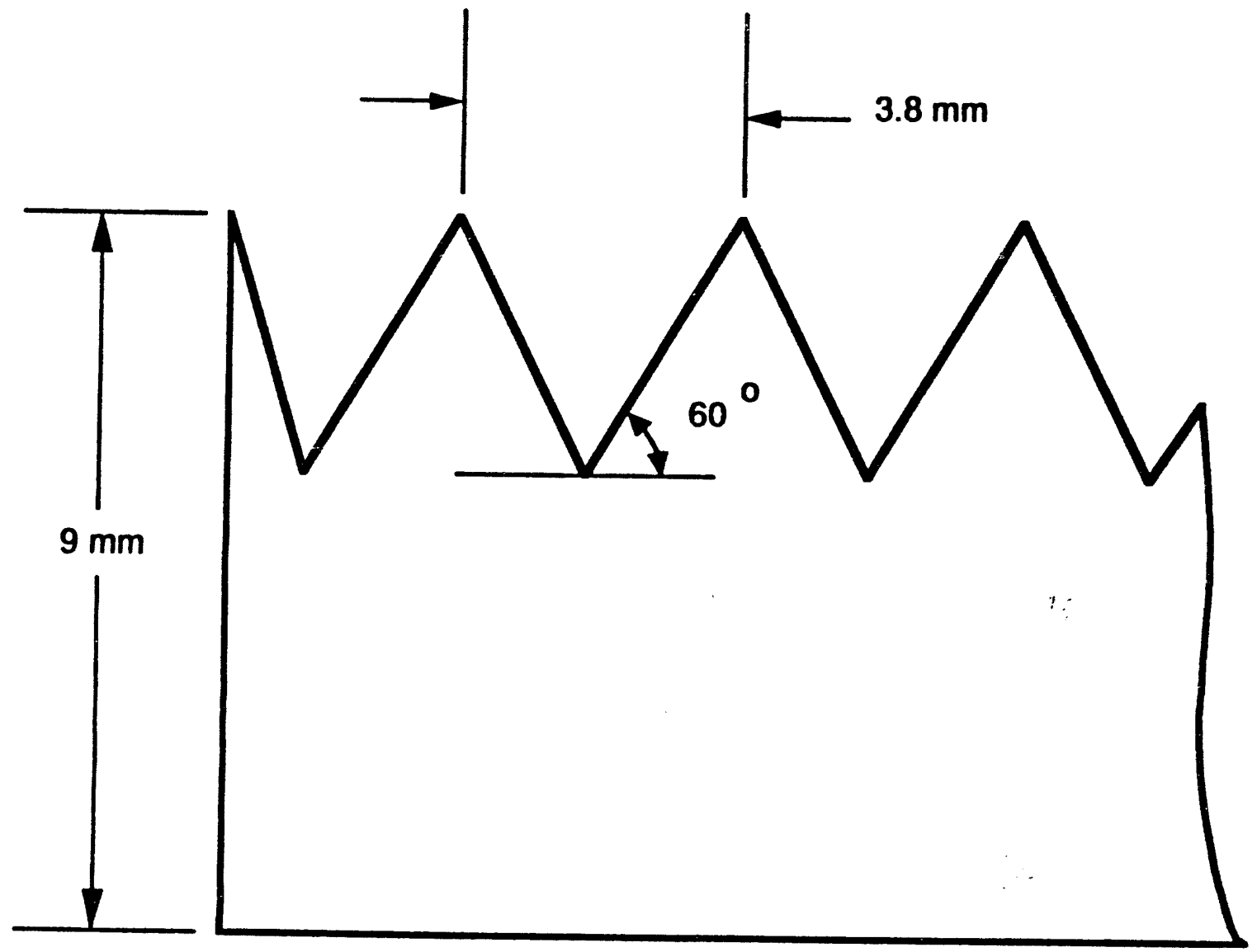

8917437

Figure 1-2. Details of the Corrugated Collector Plate Dimensions. 


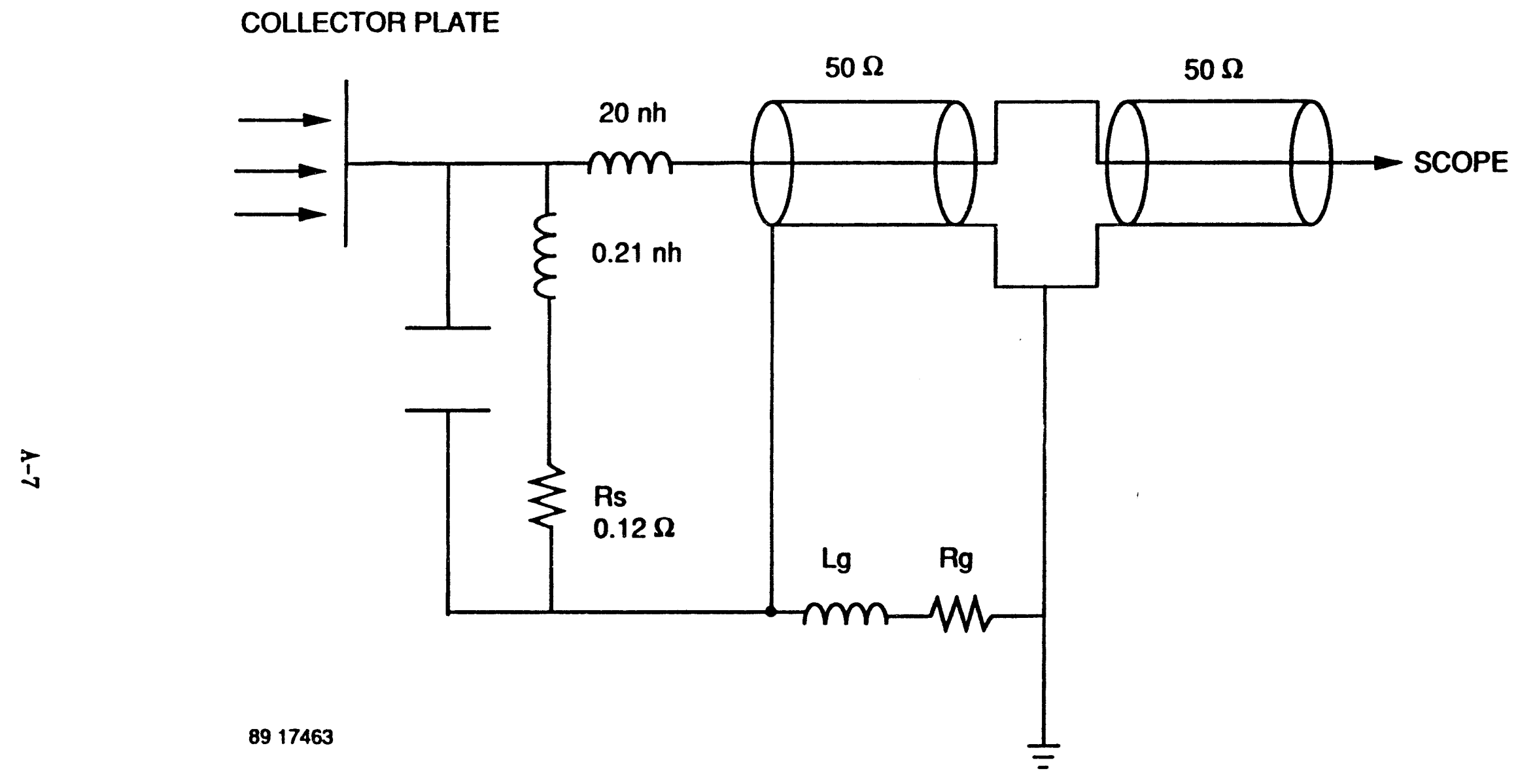

Figure 1-3. Equivalent Electrical Schematic for the Faraday Cup then Connected to an Oscilloscope. 


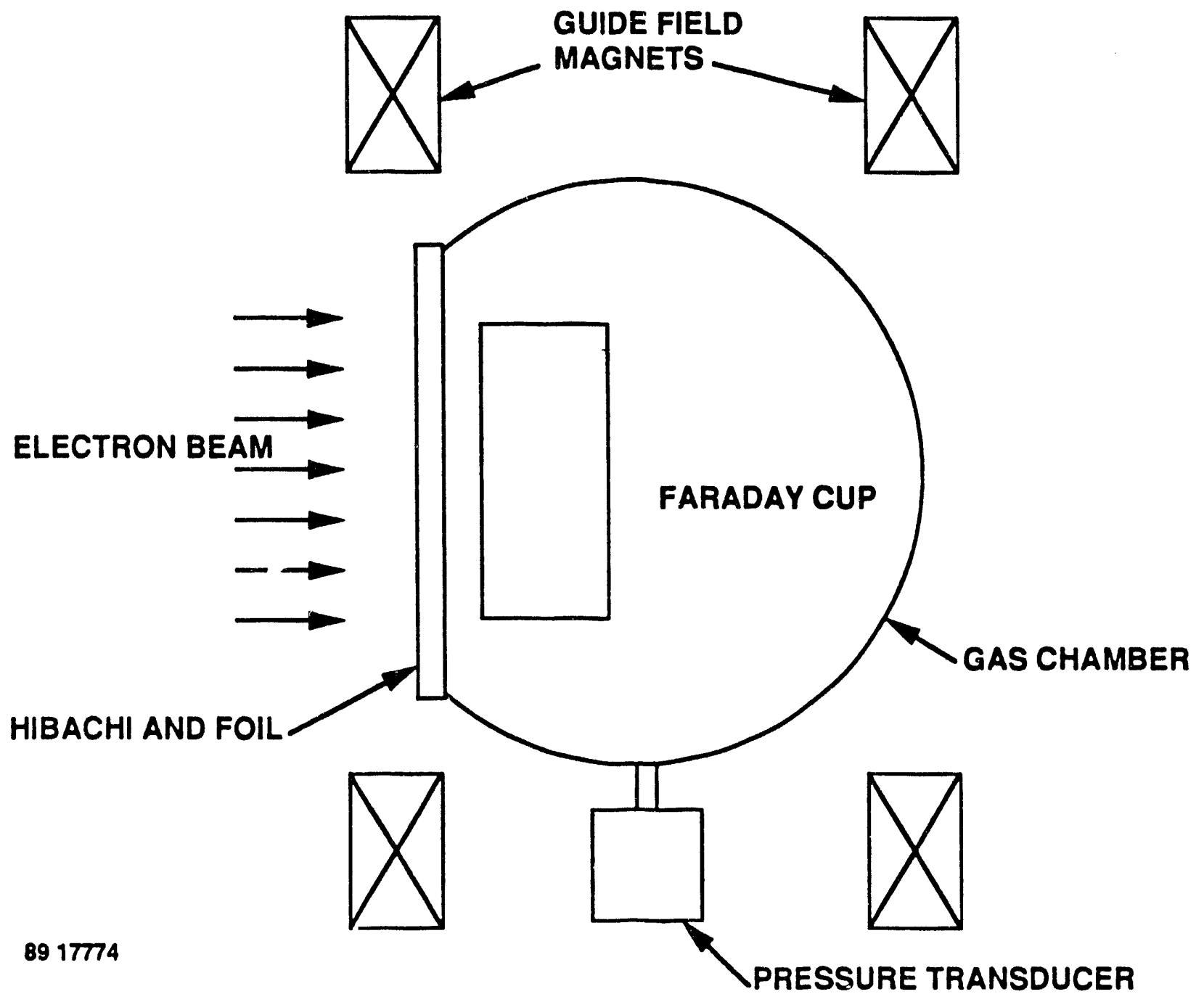

Figure 1-4. Cross-section of the Tahoma Laser Chamber Showing the Typical Location for the Faraday Cup. 
in a vacuum. The results are shown in Figure $1-5$, which plots the peak current measured by the cup for various collectors. The values are relative to the beryllium value that is assumed to have a $2 \%$ absolute electron albedo as determines by Wright and Trump. (A-1) Also plotted in Figure $A-5$ is a curve representing the Wright and Trump results assuming $400 \mathrm{keV}$ electrons. The data from our Faraday cup are in excellent agreement with the Wright and Trump curve.

Assuming that beryllium backscatters $2 \%$ of the oncoming $400 \mathrm{keV}$ electrons, then the data in Figure A-5 indicate the following electron albedos for the various collector plate materials: Beryllium $=2 \%$, Carbon $=3 \%$, Aluminum $=9 \%$, and Lead $=45 \%$. Since carbon has essentially the same albedo as beryllium, but is much easier to handle, it is the preferred collector material. The high albedo of lead demonstrates why this material is effective as an electron back reflector in the beam stop.

The effect of corrugating the face plate surface (i.e. Figure 1-2) to improve the electron collection efficiency is given in Figure A-6. Shown are the oscilloscope traces of the Faraday cup signal (bottom trace) and the current through the e-beam diode (top trace) as measured using a $\mathrm{dB} / \mathrm{dt}$ probe inside the e-beam diode vacuum chamber. Note that the corrugated carbon [Figure A-6(a)] gives a higher signal than the corrugated aluminum [Figure $A-6(b)$ ] and that the corrugated aluminum has only a slightly higher signal than the flat aluminum face plate [Figure A-6(c)]. This demonstrates that at least for low $\mathrm{Z}$ materials, there is little improvement in electron collection efficiency by corrugating the face plate surface.

As a result of these initial comparison tests, carbon was selected as the best material to use and all subsequent measurements with the cup used the corrugated carbon face plate. (Although corrugation probably provides negligible benefit with carbon, since a flat carbon face plate was not available, the corrugated one was used.)

The linearity of the cup was verified by measuring the cup signal as a function of the geometric transmissions of various masks placed in front of the cup. The results are shown in Figure A-7. Except for a small 


\section{Z DEPENDENCE - IN VACUUM}

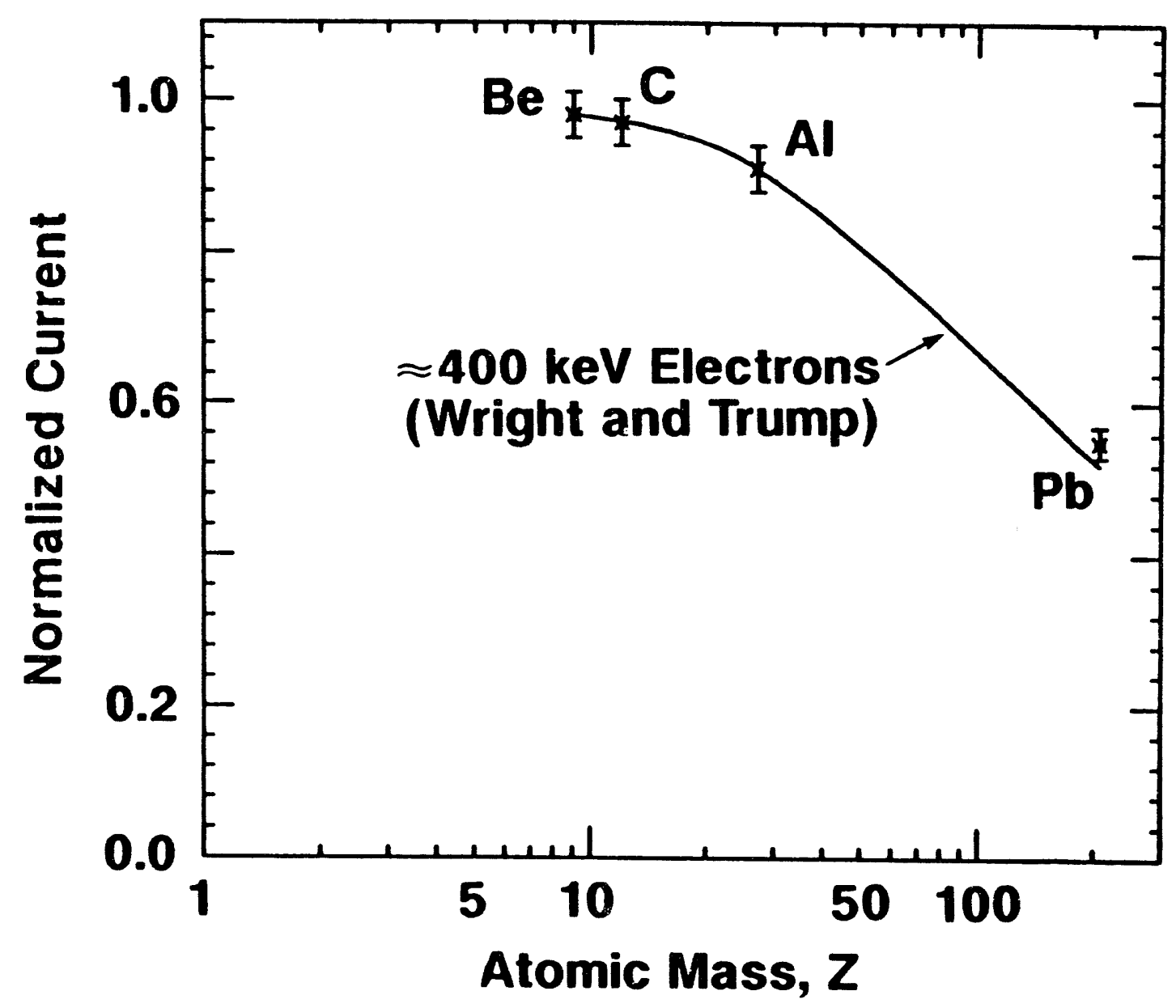

Figure 1-5. Normalized Output From the Faraday Cup as A Function of the Atomic Mass of the Collector Plate. The curve is from Reference ( $(\Lambda-1)$. 


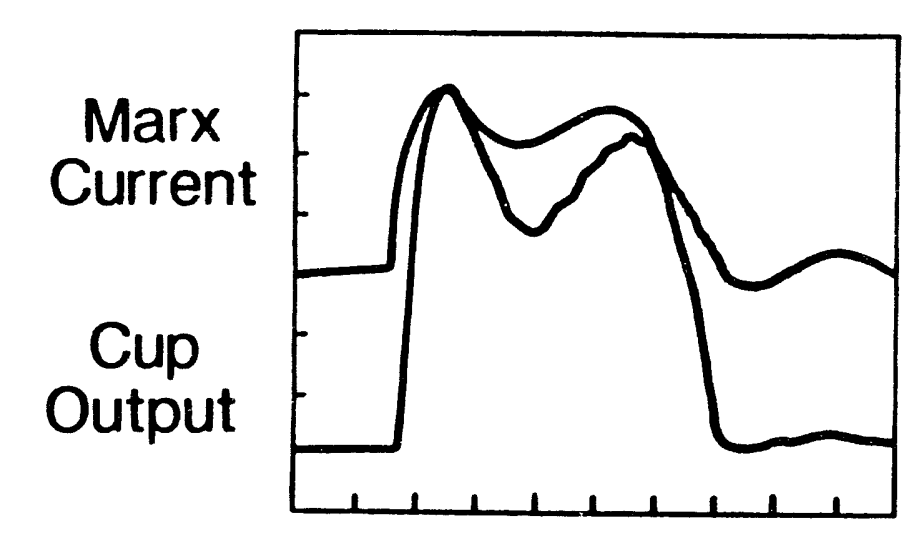

立

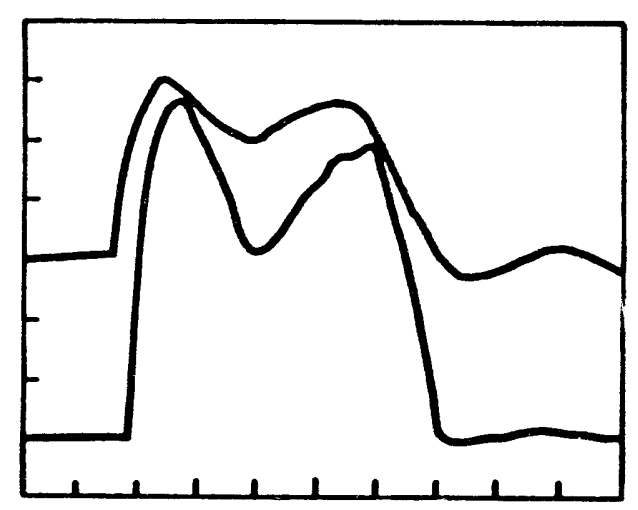

Corrugated Aluminum

(b)

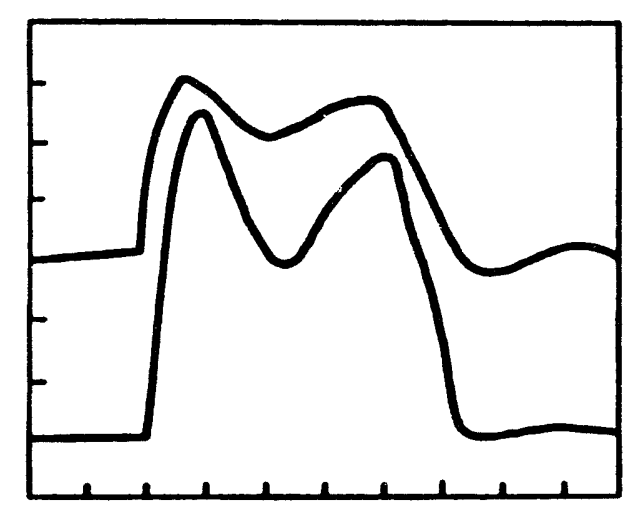

Flat Aluminum

(c)

Figure A-6. Comparison of Different Collector Plates. The top trace is the Marx current obtained from a dB/dt probe within the diode chamber; the bottom trace is the output from the Faraday Cup. Time scale is $100 \mathrm{~ns} / \mathrm{div}$. (a) For corrugated carbon. (b) For corrugated aluminum. (c) For flat aluminum. 


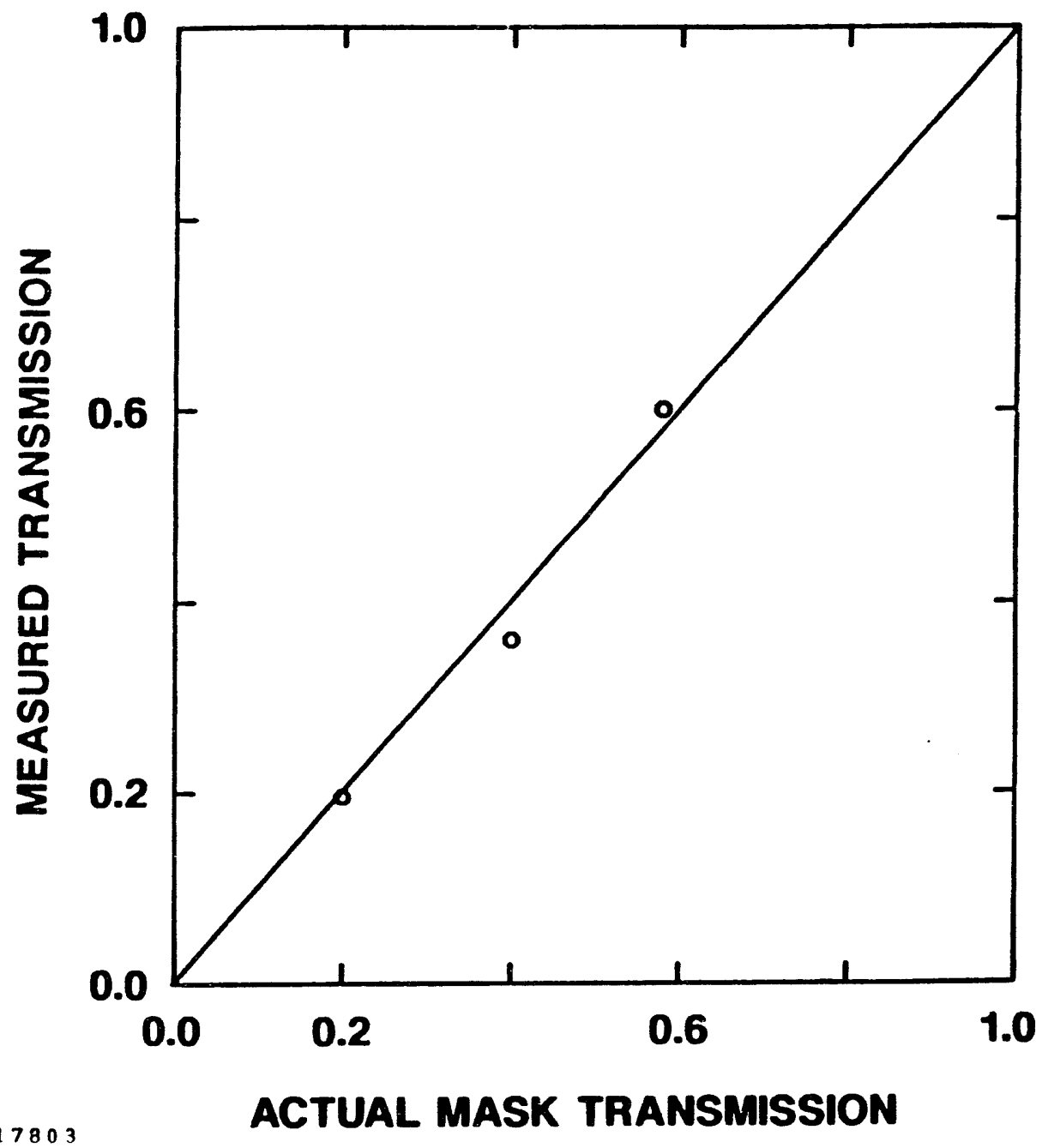

Figure A-7. Faraday Cup Linearity Check. Plotted is the cup output signal versus the geometrical transmission factor for various perforated masks placed between the e-beam and the cup. 
deviation from a straight line for the $40 \%$ mask transmission case, the data shows good linearity characteristics. (The $40 \%$ mask data may have been affected by electron loss because the thickness of the mask was comparable to the open area of the mask. Therefore, a significant portion of electrons may have been absorbed by the inner side walls of the mask.)

In order to operate the cup in a gaseous environment, it is necessary to electrically isolate the collector face plate from the grounded side walls of the cup. An e-beam impinging upon the collector plate generates a plasma in the local area that provides a conducting path from the plate to the side walls. To prevent this plasma from shorting out the plate to ground, a thin Kapton foil can be placed over the collector plate (see Figure A-1). Fortunately, at the e-beam energy used during these experiments, the thin foil $(0.025 \mathrm{~mm}$ thick) does not appreciably decrease the current density measured. This is demonstrated in Figure $1-8$, which shows the Faraday cup signal in a vacuum without the foil [Figure $\Lambda-8(a)$ ] and with the foil [Figure $A-8(b)$ ]. There is essentially no difference between the two waveforms.

The effectiveness of the foil can be seen in Figure A-8. This shows the Faraday cup signal when operating in 1000 Torr of a $10 \% \mathrm{Kr} / 80 \% \mathrm{Ar}$ gas mixture. Notice that without the foil [Figure $A-\theta(a)$ ] the cup signal is roughly $40 \%$ lower than with the foil [Figure $A-\theta(b)$ ].

Comparisons of the current density measured with the foil for vacuum and air in the laser chamber are depicted in Figure A-10. The data points represent the peak value of the signals detected by the cup, and were obtained by moving the cup away from the foil. As can be seen, the current densities are comparable for both cases, except $25 \mathrm{~cm}$ from the foil where the signal starts to significantly decrease in air as compared to vacuum.

Figure A-11 shows the same measurement this time comparing air to a 1000 Torr $10 \% \mathrm{Kr} / 80 \%$ Ar (typical $\mathrm{KrF}$ mixture). The higher stopping power of $\mathrm{Ar}$ and $\mathrm{Kr}$ results in a significant attenuation of the e-beam as it traverses through the gas. 


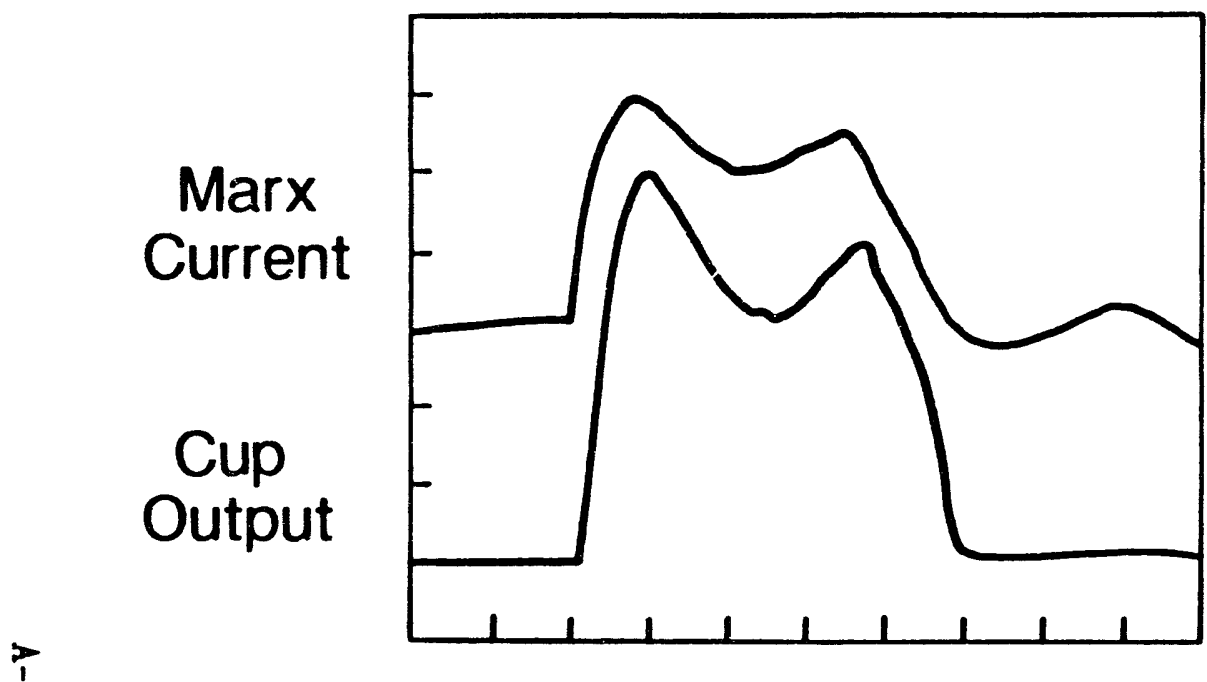

ค9 17804

\section{Without Foil}

(a)

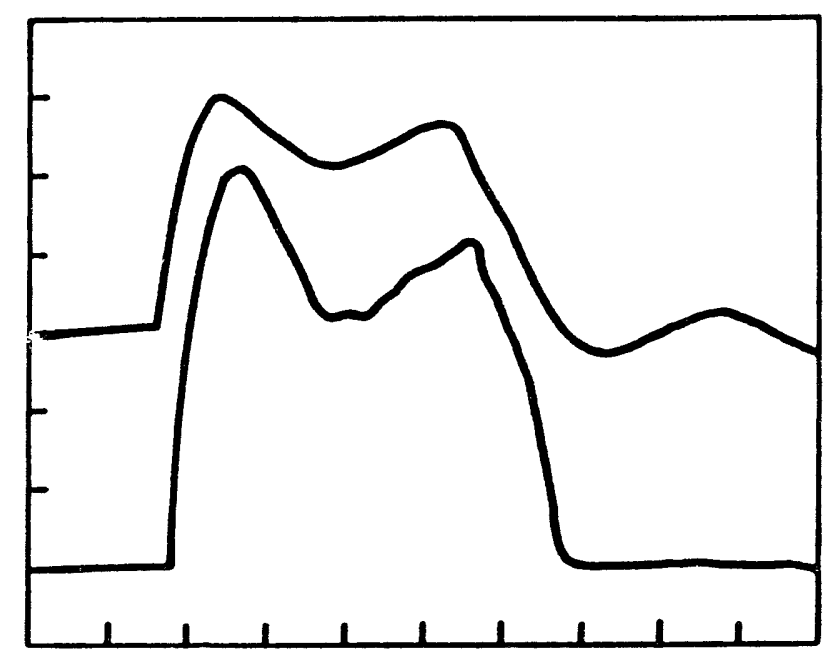

With Foil

(b)

Figure A-8. Relative Effect of $0.025 \mathrm{~mm}$ Kapton Foil Over Collector Plate. The top trace is the Marx current obtained from a dB/dt probe within the diode chamber; the bottom trace is the output from the Faraday Cup. Time scale is $100 \mathrm{~ns} / \mathrm{div}$. The carbon face plate is used under vacuum conditions. (a) Without the foil. (b) With the foil. 


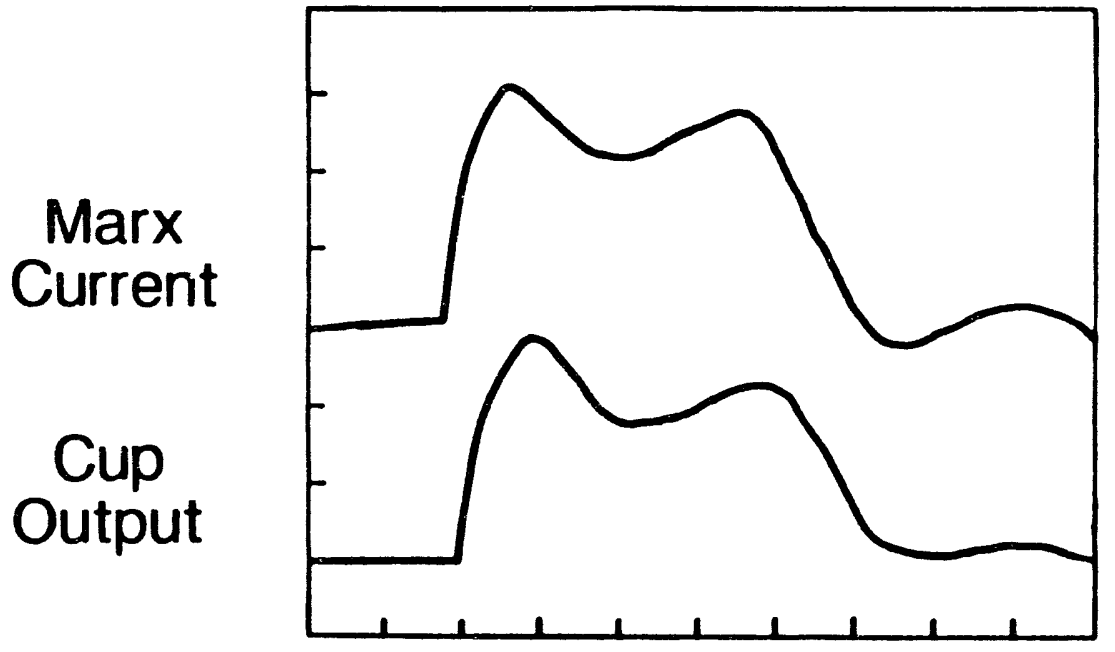

Without Foil

(a)

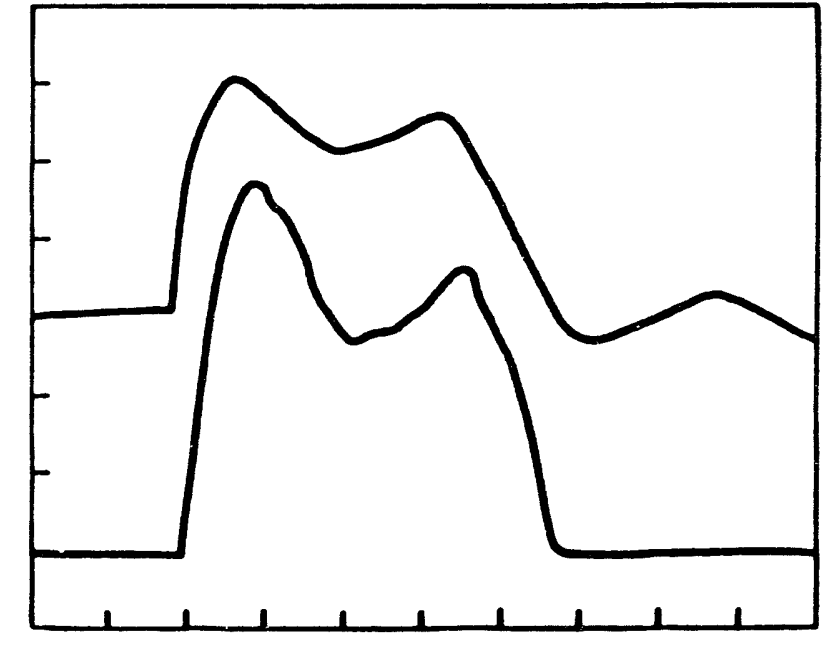

With Foil

(b)

Figure 1-9. Performance of the Faraday Cup in a Gas Mixture (10\% $\mathrm{Kr} / 90 \% \mathrm{Ar}$ at 1000 Torr). The top trace is the Marx current obtained from a $\mathrm{dB} / \mathrm{dt}$ probe within the diode chamber; the bottom trace is the output from the Faraday Cup. Time scale is $100 \mathrm{~ns} / \mathrm{div}$.
(a) Without the Kapton foil.
(b) With the Kapton foil. 


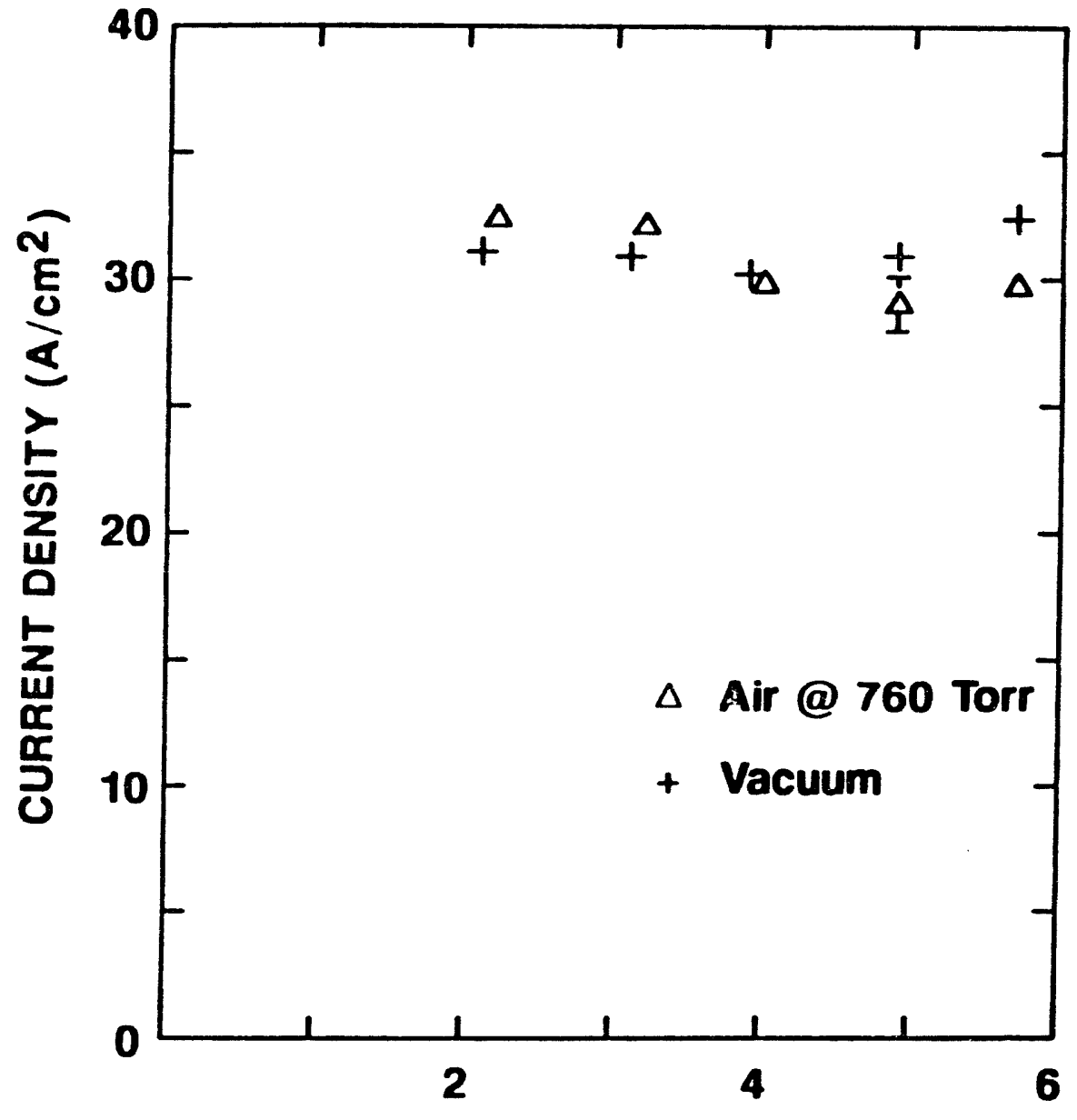

8917806

\section{DISTANCE FROM FOIL $(\mathrm{cm})$}

Figure A-10. Comparison of Current Density With Either Vacuum or Air in the Laser Chamber. The carbon face plate and Kapton foil are used. 


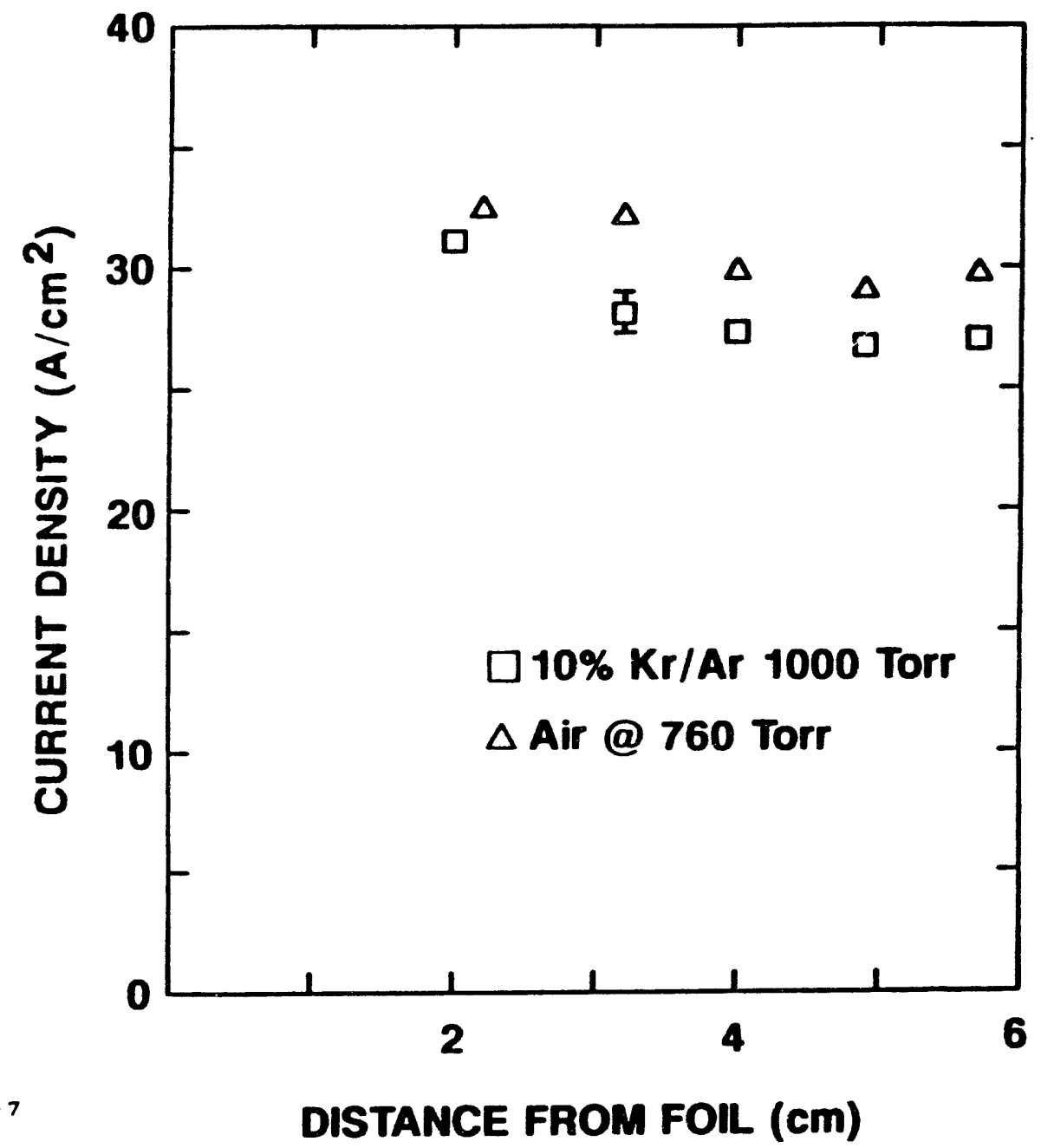

Figure A-11. Comparison of Current Density With Bither Air or a $\mathbf{K r} / \mathbf{A r}$ Mixture in the Laser Chamber. The carbon face plate and Kapton foil are used. 
Proper grounding of the cup is critical to ensure that misleading signals are not obtained. Several grounding configurations were investigated. These include grounding the cup by sitting it on a base plate that is in contact with the grounded laser chamber or bolting a copper strap directly to the cup from the grounded chamber. At the opposite extreme, the cup was electrically floated in either air or vacuum to see the effects of improper grounding. The results are depicted in Figure A-12. Figure A-12(a) shows the signal during proper grounding conditions in air. If this ground connection is lifted [Figure $\mathrm{A-12(b)}$ ], then the cup signal towards the end of the pulse begins to deviate from its normal value. In fact, a "tail" can be observed in the cup signal after termination of the main pu se. The situation becomes much worse if the cup is operated in a vacuum without a proper ground connection [Figure A$12(c)]$.

The Kapton foil over the collector plate, if also sealed to the side walls, can permit different gases to be used inside the cup. In particular, electron attaching gases such as $\mathrm{SF}_{6}$ can be used. The purpose of the attacher gas would be to remove low energy electrons created around the collector plate area from the primary e-beam. If large in number, these secondary electrons might appear as an extraneous signal on the cup.

The cup was tested with either 10 or 60 Torr of $\mathrm{SF}_{6}$ inside the cup with vacuum on the outside. A $0.013 \mathrm{~mm}$ Kapton foil was used and the gas depth was $<1 \mathrm{~cm}$. A $<5 \%$ reduction in signal was observed for the 60 Torr test, and a negligible effect was measured for the 10 Torr case. The 5\% reduction is probably due to the significant electron stopping power of $\mathrm{SF}_{6}$ and not its attachment properties. These measurements seem to indicate that there is not a significant amount of secondary electrons being generated near the collector plate.

Finally, measurements were performed with a Pearson probe located in back of the cup to measure the current through the output lead of the cup. This is an alternative way of sensing the current that does not use the resistors inside the cup. The Pearson probe gave the same current readings and the Faraday cup. 


\section{2x Higher Signal}

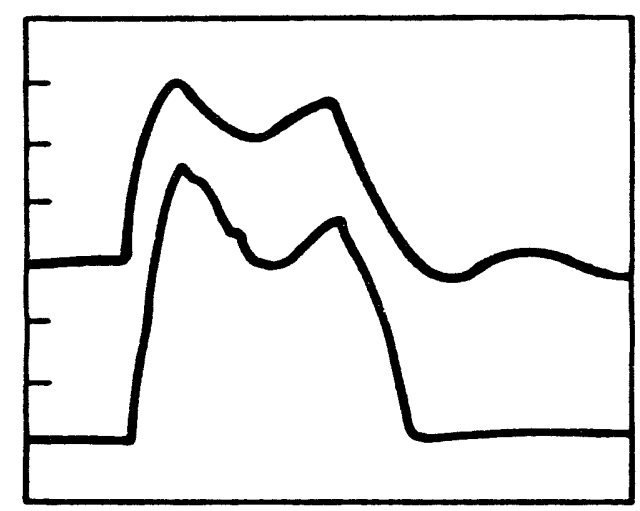

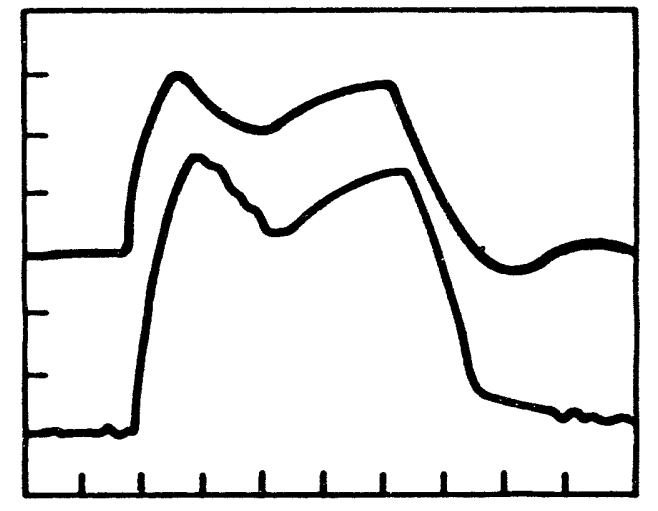

760 Torr Air

Floated

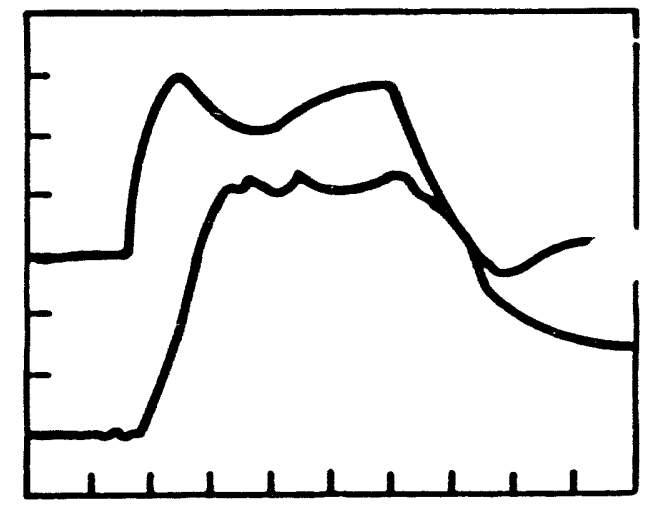

Vacuum Floated

Figure 1-12. Performance of Faraday Cup During Different Grounding

Conditions. The top trace is the Marx current obtained from a $\mathrm{dB} / \mathrm{dt}$ probe within the diode chamber; the bottom trace is the output from the Faraday Cup. Time scale is $100 \mathrm{~ns} / \mathrm{dir}$. (a) With the cup grounded to the laser chamber in room air. (b) With the cup not grounded directly to the laser chamber in room air. (c) With the cup not grounded directly to the laser chamber in vacuum. 


\section{A.4 Conclusions}

A versatile Faraday cup has been developed and tested. It has proven to be a reliable and accurate device for measuring the e-beam current density in both vacuum and gaseous environments. Critical aspects of the cup design have been experimentally analyzed in order to understand their impact on the cup performance. This has led to an improved understanding of the cup design and performance capabilities.

\section{A.5 References}

A-1. K.A. Wright and J.G. Trump, "Back-Scattering of Megavolt Electrons From Thick Targets," J. Appl. Phys. 33, 687 (1962). 


\section{Appendix B}

\section{B-BEAN DEPOSITION CALCULATION}

FOR NONUNIFORY TBMPERATURE DISTRIBUTION

\section{B.1 Introduction}

Calculating energy deposition from pressure jump data typically involves only the initial thermodynamic state of the gas. A temporal extrapolation of the pressure waveform to $t=0=t_{0}$ is made and then scaled by the volume dilution ratio defined as the total volume divided by the pumped volume. This effective pressure rise at $t_{0}$ is correlated to a temperature rise (and consequently energy) by the simple expression,

$$
\Delta T / T=\Delta P_{\text {eff }} / P
$$

Generally, the thermodynamic states of the pumped and unpumped gas regions are ignored throughout the temporal history of the pressure jump. This is equivalent to assuming that the temperature of the entire gas chamber is uniformly changing in time. In the discussion that follows, a simple thermodynamic model is presented that explores the potential nonuniformity of temperature in the gas chamber at the time that pressure equilibration is reached (typically $t_{0}+10 \mathrm{~ms}$ to $100 \mathrm{~ms}$ ). Although the calculated increase in the initial temperature $(\Delta T)$ are the same for both cases, quantifying the intermediate states may aid in estimating transient heat losses due to collisions with chamber walls during the pressure rise.

\section{B.2 Yode1}

Figure B-1(a) through B-1(c) show cross sections of two concentric cylindrical volumes representing three stages in the thermodynamic history of the pumped and unpumped volumes. Symbol definitions are also given. The pressure transducer is shown connected to the outer circle implying that the pressure is measured globally over the total volume. The inner 

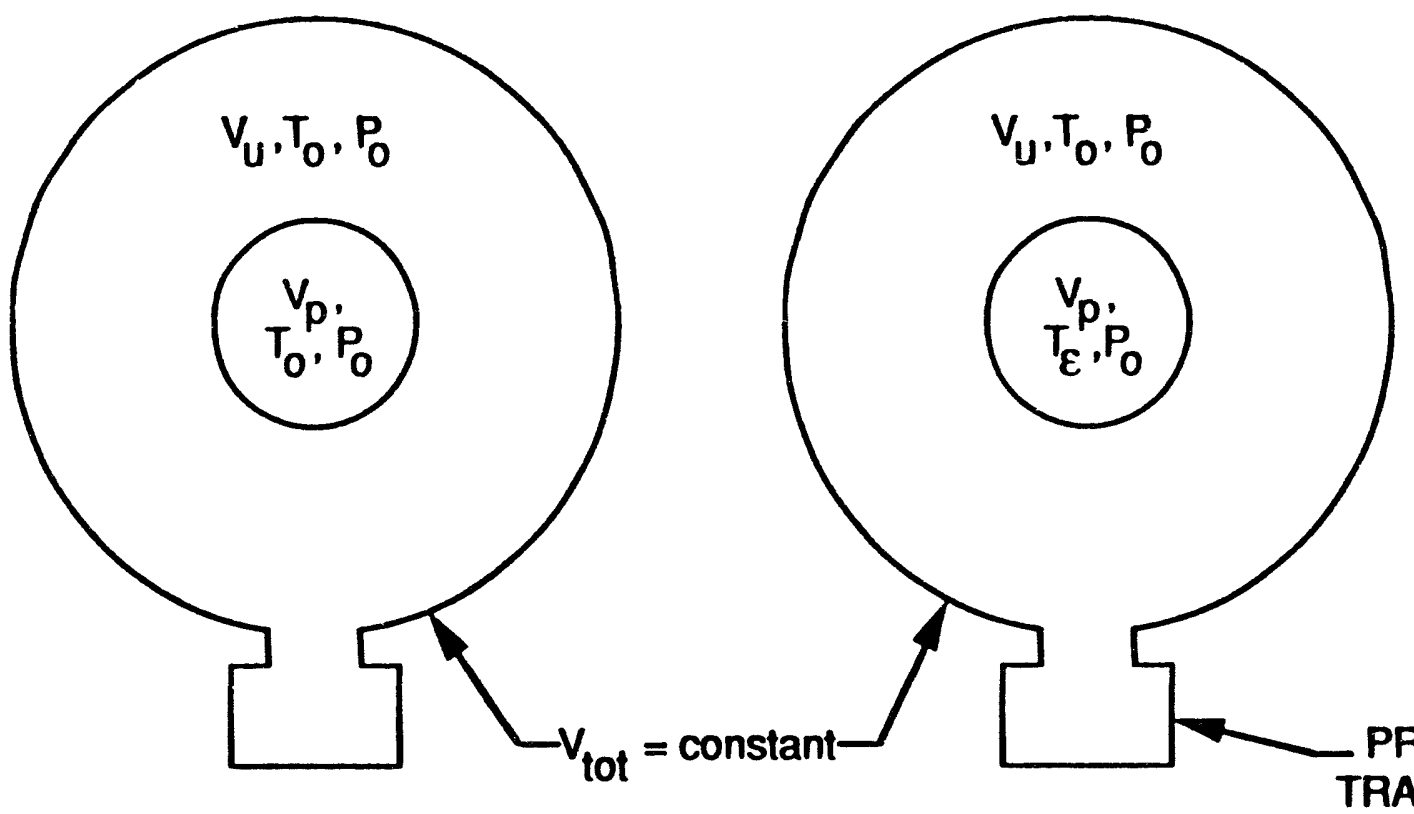

$t<0$, Before e-beam

$V_{p}=$ Pumped Volume

$V_{u}=$ Unpumped Volume

$T_{0}=$ Initial Temperature

$P_{0}=$ Initial Pressure

\section{$t=\varepsilon$, End of $\theta$-beam \\ $T_{\varepsilon}=$ Pumped Volume Temperature}

PRESSURE

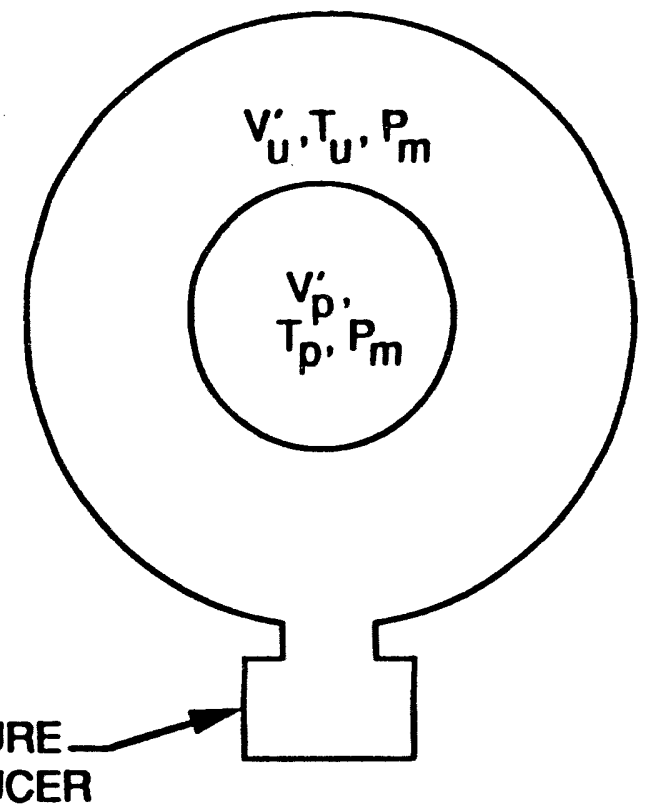

$t=$ Equilibration

$V_{p}^{\prime}=$ Pumped Volume After Expansion

$V_{u}^{\prime}=$ Unpumped Volume After Compression

$T_{u}=$ Unpumped Volume Temperature

$T_{p}=$ Pumped Volume Temperature

$P_{m}=$ Measured Pressure

(a) 
circle represents the pumped region. Figure B-1(a) shows the initial conditions before the e-beam excitation $(t<0) . V_{p}$ (pumped volume) and $V_{\text {tot }}$ (total volume) are known, and $V_{u}$ (unpumped volume) $=V_{\text {tot }}-V_{p}$. The pressure and temperature, $P_{0}$ and $T_{0}$, are uniform throughout the total chamber volume.

Figure B-1(b) shows the system immediately at the end of the e-beam pulse $(t=\epsilon)$. Deposition times are typically fast enough to ignore gas motion during the pulse so that, as diagramed, the only change to the system is the increase in temperature of the pumping volume.

Figure B-1(c) shows the system at the time of pressure equilibration. Ideally, the pumped volume expands uniformly which decreases the gas density and cools as work is done to compress the colder gas in the unpumped region. Expansion and compression stop at the equilibration time. Temperatures and volumes are calculated for this case, after which the temperature rise $\mathrm{T}_{\epsilon}$ due to the e-beam deposition is found.

\section{B.3 Analysis}

The analysis assumes negligible transient losses and mixing. The thermodynamics appear to be equivalent to the classical piston problem, and consequently are assumed to be reversibly adiabatic. The equations of state for an ideal gas are: ${ }^{\mathrm{B}-1}$

$$
\begin{aligned}
& \mathrm{Tv}^{\gamma-1}=\text { const. } \\
& \mathrm{Tp}^{\frac{1-\gamma}{\gamma}}=\text { const. } \\
& \mathrm{pv}^{\gamma}=\text { const. } \\
& \gamma=\mathrm{C}_{\mathrm{p}} / \mathrm{C}_{\mathrm{v}} .
\end{aligned}
$$


Also in an adiabatic process, work done by a part of the system on another equals the change in internal energy of the part that the work is done on,

$$
W=\Delta U
$$

where,

$$
\begin{aligned}
& W=\frac{1}{1-\gamma}\left(p_{2} v_{2}-p_{1} p_{1}\right) \\
& \Delta U=\left\langle M C_{v}\right\rangle T
\end{aligned}
$$

and the quantity $\left\langle\mathrm{MC}_{\mathbf{v}}\right\}$ is defined as

$$
\sum_{1} m_{i} C_{v_{i}} \text {, where } i=K r, A r \text {, or } F_{2}
$$

At the equilibration time, the total energy deposited into the gas $(\Delta Q)$ is,

$$
\Delta Q=\Delta U_{\text {tot }}
$$

where now $\Delta U_{\text {tot }}=\Delta U_{p}+\Delta U_{u}$. The subscripts $p$ and $u$ always refer to the pumped and unpumped regions, respectively. Applying Equation [B-5] directly,

$$
\begin{aligned}
& \Delta Q=\left\langle\underline{u}_{p} C_{v}\right\}\left(T_{\epsilon}-T_{0}\right) \\
& \Delta U_{p}=\left\langle u_{p} C_{v}\right\}\left(T_{p}-T_{0}\right) \\
& \Delta U_{u}=\left\langle u_{u} C_{v}\right\}\left(T_{u}-T_{0}\right)
\end{aligned}
$$

Solving for $T_{\epsilon}$ and $\Delta Q$, and eliminating $\mathbb{M}_{u} / \mathbb{M}_{p}$ by using the volume dilution ratio, $R=v_{\text {tot }} / V_{p}=u_{\text {tot }} / M_{p}$ where $u_{\text {tot }}=u_{p}+u_{u}$, then

$$
\begin{gathered}
T_{\epsilon}=T_{p}+(R-1)\left(T_{u}-T_{0}\right) \\
\Delta Q=\left\langle\underline{U}_{\text {tot }} C_{v}\right\}(1 / R)\left(T_{\epsilon}-T_{0}\right) .
\end{gathered}
$$


The equilibration temperatures $T_{p}$ and $T_{u}$ must now be found. $T_{u}$ is obtained by utilizing Equation [B-2] for the two conditions in Figures B-1(a) and B$1(c)$.

$$
\mathrm{T}_{u}=\left(\mathrm{P}_{\mathrm{m}} / \mathrm{P}_{0}\right)^{\frac{\gamma-1}{\gamma}} \mathrm{T}_{0}
$$

where $P_{m}=$ measured equilibration pressure.

$T_{p}$ is found after several steps. $V_{p}$ ' and $V_{u}$ ' are the expanded pumped and compressed unpumped volumes at the pressure equilibration time, and are related by $v_{p}^{\prime}=v_{\text {tot }}-v_{u}{ }^{\prime}$. From Equations [B-4] and [B-5], and using $\left\langle\mathrm{M}_{\mathrm{p}} \mathrm{C}_{\mathrm{v}}\right\rangle\left(\mathrm{T}_{\mathrm{p}}-\mathrm{T}_{0}\right)=[1 /(1-\gamma)]\left(\mathrm{P}_{\mathrm{m}} \mathrm{V}_{\mathrm{p}}^{\prime}-\mathrm{P}_{0} \mathrm{~V}_{\mathrm{p}}\right)$, we find

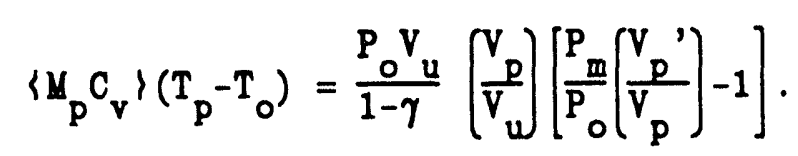

Similarly, $\left\langle M_{u} C_{v}\right\}\left(T_{u}-T_{o}\right)=[1 /(1-\gamma)]\left(P_{m} V_{u}{ }^{\prime}-P_{0} V_{u}\right)$, which after solving for $v_{u}$ ' using Equation $[B-3]$ and substituting for this and $T_{u}$ yields the relation,

$$
\left\langle M_{u} C_{v}\right\rangle T_{0}=P_{0} V_{u} /(1-\gamma)
$$

Using this and Equation [B-8],

$$
T_{p}=\left(P_{m} / P_{0}\right)\left(V_{p}^{\prime} / V_{p}\right) T_{0}
$$

Now substituting for $v_{p}$ ',

$$
T_{p}=\left[\left(\frac{P_{m}}{P_{0}}\right) R-\left(\frac{P_{m}}{P_{0}}\right)^{\frac{\gamma-1}{\gamma}}(R-1)\right] T_{0} .
$$

To find $T_{\epsilon}, T_{p}$ and $T_{u}$ are substituted into Equation [B-6] yielding,

$$
T_{\epsilon}=R\left(\Delta P / P_{0}\right) T_{0}+T_{0}
$$


Hence,

$$
\left(T_{\epsilon}-T_{0}\right) / T_{0} \equiv \Delta T / T_{0}=R\left(\Delta P / P_{0}\right)
$$

and

$$
\Delta Q=\left\langle\underline{u}_{\text {tot }} C_{\mathbf{v}}\right\rangle\left(\Delta \mathrm{P} / \mathrm{P}_{0}\right) \mathrm{T}_{0}
$$

For the experiments performed on Tahoma, the appropriate values for the parameters in Equation [B-9] can be substituted to give the final expression for the energy deposition as a function of the pressure jump. Recalling that

$$
\left\langle\underline{u}_{\text {tot }} C_{v}\right\}=\sum_{1} m_{i} C_{v_{i}}
$$

and that $m_{i}=\left(P_{i} / 760\right.$ torr $)\left(V_{\text {tot }} / 22.41\right)\left(273 / T_{0}\right) A$, where $A=$ atomic mass of the gas. Then, for all $\mathrm{Kr} / \mathrm{Ar}$ mixes during the Tahoma experiments, we find that,

$$
\Delta Q(J)=2.77 \Delta P(\text { Torr })
$$

\section{B.4 References}

B-1. F.W. Sears, An Introduction to Thermodynamics, the Kinetic Theory of Gases, and Statistical Mechanics, (Addison-Wesley, Reading, 1953), p. $67-70$. 
